\title{
Proteogenomic characterization and mapping of nucleosomes decoded by Brd and HP1 proteins
}

\author{
Gary LeRoy ${ }^{1 \dagger}$, louri Chepelev²+, Peter A DiMaggio ${ }^{1}$, Mario A Blanco ${ }^{1}$, Barry M Zee ${ }^{1,3}$, Keji Zhao ${ }^{2}$ and \\ Benjamin A Garcia ${ }^{1,3,4,5^{*}}$
}

\begin{abstract}
Background: Histone post-translational modifications (PTMs) constitute a branch of epigenetic mechanisms that can control the expression of eukaryotic genes in a heritable manner. Recent studies have identified several PTMbinding proteins containing diverse specialized domains whose recognition of specific PTM sites leads to gene activation or repression. Here, we present a high-throughput proteogenomic platform designed to characterize the nucleosomal make-up of chromatin enriched with a set of histone PTM binding proteins known as histone PTM readers. We support our findings with gene expression data correlating to PTM distribution.

Results: We isolated human mononucleosomes bound by the bromodomain-containing proteins $\mathrm{Brd} 2$, $\mathrm{Brd} 3$ and Brd4, and by the chromodomain-containing heterochromatin proteins HP1 $\beta$ and HP1 $\alpha$. Histone PTMs were quantified by mass spectrometry (ChIP-qMS), and their associated DNAs were mapped using deep sequencing. Our results reveal that Brd- and HP1-bound nucleosomes are enriched in histone PTMs consistent with actively transcribed euchromatin and silent heterochromatin, respectively. Data collected using RNA-Seq show that Brdbound sites correlate with highly expressed genes. In particular, $\mathrm{Brd} 3$ and $\mathrm{Brd} 4$ are most enriched on nucleosomes located within HOX gene clusters, whose expression is reduced upon Brd4 depletion by short hairpin RNA.

Conclusions: Proteogenomic mapping of histone PTM readers, alongside the characterization of their local chromatin environments and transcriptional information, should prove useful for determining how histone PTMs are bound by these readers and how they contribute to distinct transcriptional states.
\end{abstract}

\section{Background}

A cell's transcriptional program is governed not only by cis-acting DNA sequences, but also by chromatin structure, DNA methylation and histone post-translational modifications (PTMs). Chromatin modifications and the gene expression patterns that accompany them are maintained when a cell divides and are thus known as 'epigenetic' $[1,2]$. Histone lysine methylation is associated with both transcribed and non-transcribed chromatin. For example, H3K4me3 is found in the 5 ' region of active genes, whereas H3K36me2 and H3K36me3 are enriched within the body and the 3 ' end of active genes, respectively $[3,4]$. However, H3K9 and H3K27 trimethylation are implicated in both

\footnotetext{
* Correspondence: bagarcia@princeton.edu

† Contributed equally

'Department of Molecular Biology, Princeton University, 415 Schultz Laboratory, Princeton NJ 08544, USA

Full list of author information is available at the end of the article
}

the formation and spreading of constitutive heterochromatin and silencing of euchromatin. In contrast to methylation, histone $\mathrm{H} 3$ and $\mathrm{H} 4$ acetylation is a general characteristic of transcriptionally active chromatin. Depending on the residue modified, acetylations can be localized to nucleosomes near the $5^{\prime}$ promoter region or enriched throughout coding regions of actively transcribed genes. For instance, chromatin immunoprecipitation experiments have shown that H3K27ac and H3K14ac are enriched on only a few nucleosomes at the promoters of active genes, whereas $\mathrm{H} 4$ acetylations correlate with large euchromatic regions containing transcribed loci $[3,5]$.

The 'histone code' hypothesis proposes that specific histone PTMs encode regulatory information that is read by the binding of accessory proteins. These accessory proteins, termed 'readers', bind via specialized histone-PTMbinding domains such as bromodomains, chromodomains and plant homeodomains (PHDs) [1]. The bromodomain binds directly to acetylated lysines in histones and is

\section{(Ciomed Central}


commonly found in proteins associated with gene activation, such as Brg1, a subunit of the hSWI/SNF remodeling complex, the acetyltransferase hGcn5, and the Brd proteins, which are mammalian homologs of the Drosophila trithorax Fsh1 and Fsh2 proteins [6]. The Brd2, Brd3 and Brd4 proteins are referred to as BET proteins as they contain tandem bromodomains and an extraterminal domain of unknown function [7]. In vitro studies have demonstrated that Brd2 and Brd3 possess nucleosome chaperone activities that allow RNA polymerase II to elongate transcripts through hyperacetylated nucleosomes, implicating their direct role in transcription [8]. Several reports have also implicated Brd regulation of cell cycle progression and inflammatory response $[9,10]$. Thus, the unique protein architecture and function of Brd proteins qualify them as promising targets for selective pharmacological inhibitors such as immunosuppressants and anticancer drugs. In fact, recent publications have reported two Brd protein selective small molecule inhibitors (I-BET and JQ-1) that specifically inhibit bromodomain binding to acetylated histones, thereby blocking a lipopolysaccharideinduced cytokine storm and the growth of Brd-dependent tumors $[9,11]$.

In contrast to bromodomains, chromodomains are methyl-lysine binding domains found in proteins such as the heterochromatic proteins HP1 $\alpha$ and HP1 $1 \beta$ that bind to H3K9me3 [12]. Chromodomains are also found in the polycomb group, CBX proteins, which mediate silencing by packaging specifically methylated nucleosomes into heterochromatin-like clusters [13]. In addition to a chromodomain, HP1 $\alpha$ and HP1 $\beta$ contain a chromoshadow domain thought to be involved in interactions with other proteins [14]. The HP1 $\alpha$ and HP1 proteins form both homo- and heterodimers, and although these proteins colocalize at many heterochromatic loci, they also bind to distinct loci [15].

Here, we report experiments that elucidate the combinations of histone PTMs on nucleosomes associated with the histone code reading proteins Brd2, Brd3, Brd4, HP1 $\alpha$ and HP1 $\beta$ (Figure 1a), applying a chromatin immunoprecipitation quantitative mass spectrometry (ChIP-qMS) approach. We also utilized deep sequencing to map the DNA sequences contained within these nucleosomes to their genomic locations and used RNA sequencing and microarrays to determine the transcriptional state of the nucleosomes. Knockdown of Brd4 or HP1 $\beta$ suggests that the proper expression of many genes is dependent on their associated Brd or HP1 proteins. Providing genomic maps of where histone code readers are bound and the modifications found on such nucleosomes lays down the foundation that will aid future work aimed at deciphering how the network of chromatin-associated proteins 'translate' these histone PTMs. Additionally, these proteomic ChIP-qMS methods can be generally applied to any chromatin binding protein to characterize their local chromatin environments and identify enriched histone PTM patterns. Combined with further downstream analyses we are able to correlate this information to transcriptional states.

\section{Results}

To gain insight into the local chromatin make-up of histone PTM binding proteins, we optimized a ChIP technique to purify mononucleosomes from cells expressing tagged chromodomain- and bromodomain-containing proteins. Specifically, we generated HEK293 cell lines that express FLAG-tagged fusions of Brd2, 3, 4 and HP1 $\alpha$ and $\beta$ (Figure 1a), as these bromodomain- and chromodomaincontaining proteins are believed to mitigate opposing epigenetic functions and could provide solid proof of principle experiments. Western blots (Additional file 1) show that our transiently expressed proteins are above endogenous. As outlined in Figure 1b, following nuclear isolation and micrococcal nuclease digestion, nucleosomes bound by the various FLAG-tagged proteins were immunoprecipitated and competitively eluted with excess FLAG peptide. Figure 1c shows that FLAG-tagged Brds and HP1s all immunoprecipitate the core histones while no histones were detected from cells not expressing a FLAG-tagged protein. We find that competitive elution with FLAG peptide reduces non-specific binding compared to boiling the beads in SDS buffer. Immunoprecipitates from FLAGtagged $H P 1 \alpha$ and HP1 $\beta$ contained other abundant polypeptides, such as Tif1 $\beta$ protein, a heterochromatic protein that is known to interact and colocalize with HP1 $\alpha$ and $\beta$ $[16,17]$. Interestingly, Tif1 $\beta$ contains a bromodomain and PHD domain (Figure 1a), which may allow for a more complex histone code to be read when in complex on a nucleosome with HP1. The immunoprecipitated histones were then derivatized with propionic anhydride, trypsinized, and subjected to qMS via nano scale liquid chromatography-tandem mass spectrometry (nanoLC-MS/MS) experiments on an Orbitrap instrument [18]. The derivatization allows for the incorporation of isotopically stable $d 0$ - or $d 5$-propionyl groups such that two samples (each labeled with a different isotope) can be analyzed together in a single nanoLC-MS/MS experiment (Figure 2a) or samples can be both labeled with $d 0$-propionyl and run individually. The histone modifications found on $\mathrm{H} 3$ and H4 peptides from a ChIP-qMS and whole-genome control are then quantified using in-house developed software (see Materials and methods) as depicted in Figures 2 and 3.

\section{Characterization of PTMs on histone $\mathrm{H} 4$ from Brd- and HP1-associated nucleosomes}

PTMs observed on the $\mathrm{H} 4$ molecules from Brd-bound nucleosomes contained marks consistent with gene activation. Most striking was the enrichment in the overall 
(a)

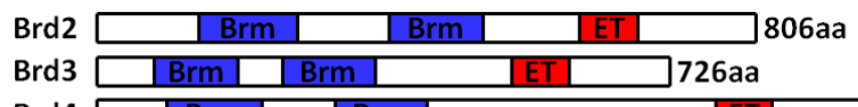

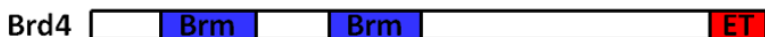

HP1 $\alpha \square$ CHD $\square$ CSHD $\square$ 191aa

HP1 1 पCHD $\square$ CSHD $\square$ 185aa

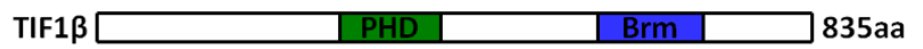

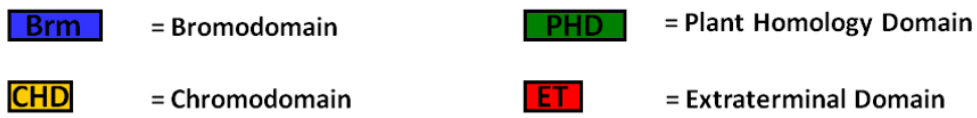

CSHD = Chromoshadow Domain

(b)

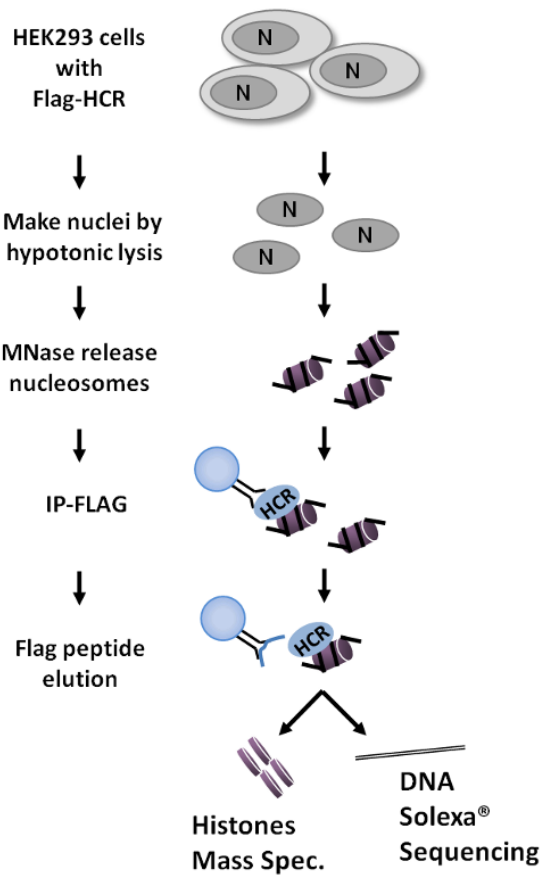

(c)
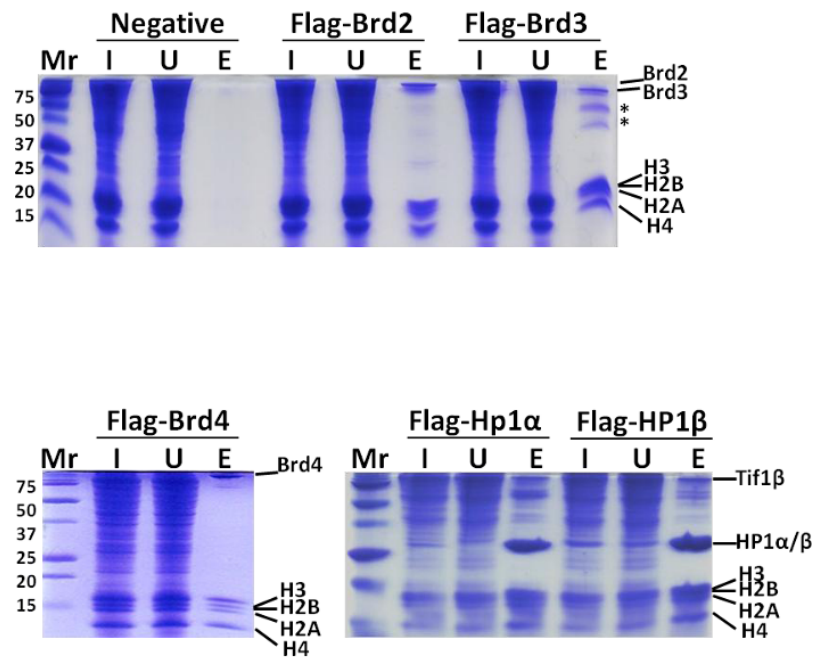

Figure 1 Isolation of Brd- and HP1-bound mononucleosomes. (a) Illustration of the domain architecture of the histone code readers. (b) Schematic of protocol used to isolate nucleosomes bound to FLAG-histone code readers (HCRs). (c) Visualization of FLAG-Brd- and FLAG-HP1bound nucleosome purifications. The proteins present in the input (I), the immunoprecipitation unbound (U), and FLAG-peptide-eluted (E), fractions were examined by SDS-PAGE and Coomassie blue staining. The asterisk denotes Brd3 breakdown products detected by liquid chromatography-tandem mass spectrometry. 
(a)

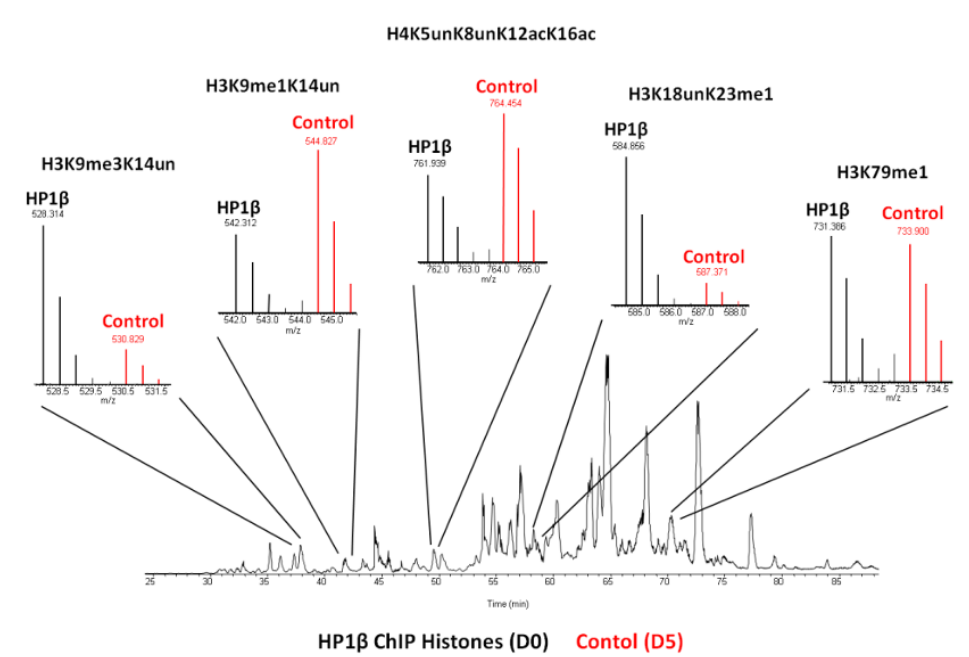

(b)

(c)

\begin{tabular}{|c|c|c|c|c|c|c|}
\hline H4 Peptide & Brd2 & Brd3 & Brd4 & $\mathrm{HP} 1 \alpha$ & HP1 $1 \beta$ & Genomic \\
\hline \multicolumn{7}{|c|}{ GKGGKGLGKGGAKR (4-17) } \\
\hline $\mathrm{H} 4(4-17)$ un & $15.12 \% \pm 1.49 \%$ & $29.35 \% \pm 1.51 \%$ & $15.58 \% \pm 2.86 \%$ & $74.12 \% \pm 3.46 \%$ & $663.31 \% \pm 3.87 \%$ & $7.46 \% \pm 2$. \\
\hline $\mathrm{H} 4(4-17) 1 \mathrm{ac}$ & $20.29 \% \pm 1.10 \%$ & $30.58 \% \pm 1.00 \%$ & & $22.65 \% \pm 2.98 \%$ & $627.63 \%$ & \\
\hline $\mathrm{H} 4(4-17) 2 \mathrm{ac}$ & $18.88 \% \pm 0.82 \%$ & $17.04 \% \pm 2.28 \%$ & $28.86 \% \pm 1.66 \%$ & $2.98 \% \pm 0.43 \%$ & $6.67 \% \pm 2.12 \%$ & $12.42 \% \pm 0.74 \%$ \\
\hline H4 $4(4-17) 3 a c$ & $24.90 \% \pm 0.44 \%$ & $16.22 \% \pm 1.60 \%$ & $22.96 \% \pm 3.04 \%$ & $0.22 \% \pm 0.04 \%$ & $6.26 \% \pm 0.04 \%$ & $2.98 \% \pm 0$ \\
\hline H4 $4(4-17) 4 a c$ & $20.81 \% \pm 2.85 \%$ & $6.80 \% \simeq 0.75 \%$ & $9.35 \% \pm 1.70 \%$ & $0.03 \% \pm 0.01 \%$ & $0.13 \% \pm 0.01 \%$ & $0.53 \% \pm 0.05 \%$ \\
\hline
\end{tabular}
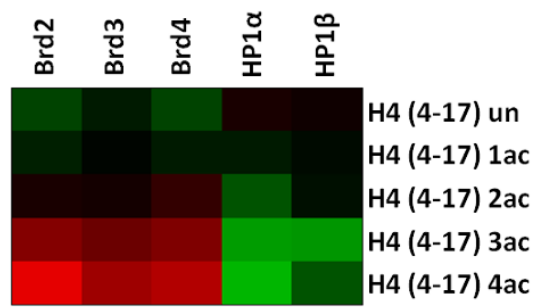

\section{ChIP/Input}

$\log _{2}$

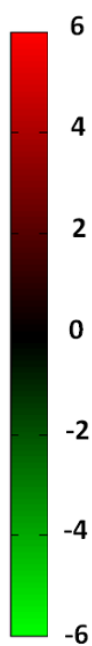

Figure 2 Histone H3 and H4 residue-specific PTMs quantified by mass spectrometry. (a) Total ion chromatogram and full MS spectrum of propionylated histone peptides used for quantification from an HP1 $\beta$ ChIP; ChIP elution D0 labeled (black), and the control ChIP input D5 labeled (red). Full MS spectrum of peptides shown; H3K9me3K14un, H3K9me1K14un, H4K5unK8unK12acK16ac, H3K18unK23me1 and H3K79me3. (b) Quantification of the degree of acetylation of the histone $\mathrm{H} 4$ peptide (amino acids 4 to 17, GKGGKGLGKGGAKR). Values in the table are the total percentage of each acetylated form and the heatmap is a representation of the fold change of the specified ChIP/Input in (log $)$ scale. (c) Heatmap depicting all modifications quantified on the histone $\mathrm{H} 3$ and $\mathrm{H} 4$ proteins. Values used to generate the heatmap are found in Additional file 2. The heatmap was generated with the fold change values of each PTM for each specified ChIP/Input in ( $\left.\log _{2}\right)$ scale. 
(a)

\section{Histone $\mathrm{H} 3$}
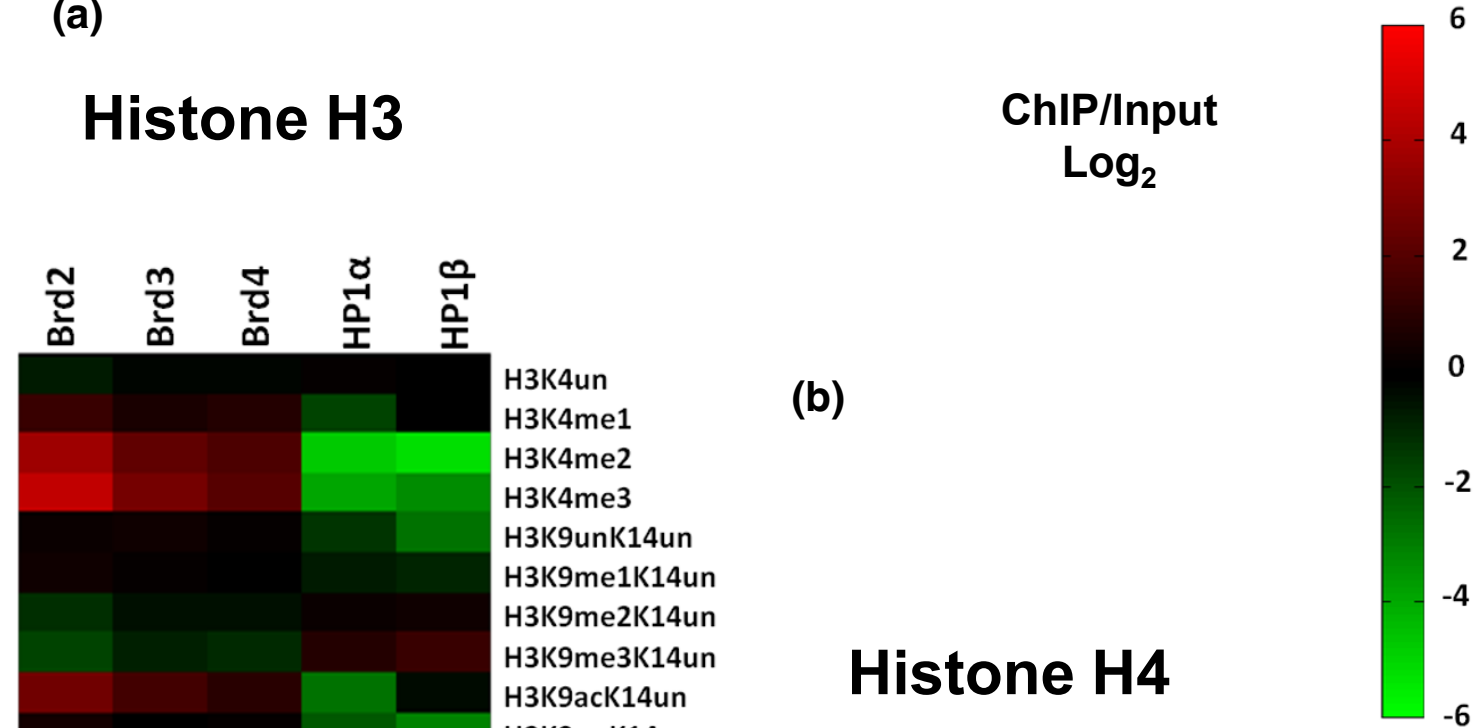

H3K9unK14ac

H3K9me1K14ac

H3K9me2K14ac

H3K9me3K14ac

H3K9acK14ac

H3K18unK23un

H3K18me1K23un

H3K18unK23me1

H3K18acK23un

H3K18unK23ac

H3K18acK23ac

H3K27unK36un

H3K27me1K36un

H3K27me2K36un

H3K27me3K36un

H3K27unK36me1

H3K27unK36me2

H3K27acK36un

H3K27me1K36me1

H3K27me1K36me2

H3K27me1K36me3

H3K27me2K36me1

H3K27me2K36me2

H3K27me3K36me1

H3K27me3K36me2

H3K79un

H3K79me1

H3K79me2
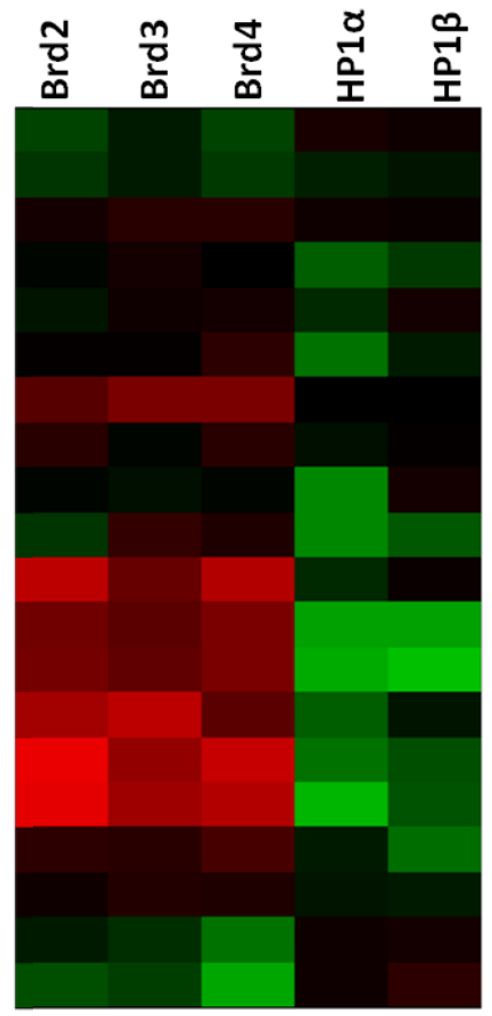

H4K5unK8unK12unK16un H4K5unK8unK12unK16ac H4K5unK8unK12acK16un H4K5unK8acK12unK16un H4K5acK8unK12unK16un H4K5unK8unK12acK16ac H4K5unK8acK12unK16ac H4K5unK8acK12acK16un H4K5acK8unK12unK16ac H4K5acK8unK12ack16un H4K5acK8acK12unK16un H4K5unK8acK12acK16ac H4K5acK8unK12ack16ac H4K5acK8acK12unK16ac H4K5ack8acK12ack16un H4K5acK8acK12acK16ac H4K20un H4K20me1 H4K2Ome2 H4K20me3

Figure 3 Quantification of PTMs on histone H3 and H4 peptides isolated with HP1 and Brd proteins. (a) Heatmap depicting all modified forms of $\mathrm{H} 3$ peptides on HP1- and Brd-bound nucleosomes by qMS. Values used to generate the heatmap are in Additional files 2 and 6 . The heatmap was generated with the fold change values for each modified H3 peptide quantified from each specified ChIP/Input in (log 2 scale. (b) Heatmap depicting all modified forms of $\mathrm{H} 4$ peptides on HP1- and Brd-bound nucleosomes by mass spectrometry. Values used to generate the heatmap are in Additional files 2 and 3. The heatmap was generated with the fold change values for each modified $\mathrm{H} 4$ peptide quantified from each specified ChIP/Input in $\left(\log _{2}\right)$ scale. 
degree of acetylation. All the potentially acetylated lysines (K5, K8, K12 and K16) occur on the same propionylated tryptic peptide, so we first quantified $\mathrm{H} 4$ tail peptides that were unmodified, mono, di-, tri- and tetra-acetylated, irrespective of the residue modified (Figure 2b, table). When compared to whole genome chromatin, all Brd nucleosomes were enriched in $\mathrm{H} 4$ acetyl modifications; 1.3 - to 2.3 -fold for the diacetylated species, 5.4- to 8.3-fold for the triacetylated species and 12.9- to 39.5-fold for the tetracetylated form (Figure 2b, heatmap). Notably, the Brd nucleosomes contained only 15 to $29 \%$ unacetylated $\mathrm{H} 4$ molecules as opposed to genomic histone $\mathrm{H} 4$, which was $50 \%$ unacetylated. As nucleosomes contain two copies of each histone, the unacetylated species within these nucleosomes is probably not directly bound by the Brds. On the other hand, HP1 $\alpha$ - and HP $1 \beta$-bound nucleosomes had relatively low levels of $\mathrm{H} 4$ acetyls. In fact, approximately $70 \%$ of the H4 tails were unmodified on HP1-bound nucleosomes (Figure 2b, table). However, the HP1 bound nucleosomes have moderate levels of $\mathrm{H} 4$ acetylation, such as tails with a monoacetyl (approximately 25\%). The single most abundant $\mathrm{H} 4$ tail acetylation in HEK 293 cells is H4K16, occurring in approximately $41 \%$ of all histone H4 molecules (Additional file 2). This particular acetylation mark was only slightly enriched on Brd nucleosomes (1.2- to 1.5-fold) (Figure 2c). We suspect that Brd proteins may not directly read this PTM; rather, its slight enrichment in Brd nucleosomes is a consequence of being associated with other active chromatin marks. Nucleosomes bound by all three Brd proteins were significantly enriched in acetylations at the other sites on histone H4; H4K5ac (4.7- to 6.3-fold), H4K8ac (4.1- to 8.7-fold) and H4K12ac (2.3- to 3.3-fold) (Figure 2c) consistent with X-ray crystallography structural studies $[19,20]$.

Our in-house software (see Materials and methods) accurately deconvoluted the mixed tandem mass spectra resulting from isobaric acetylation combinations that occur on $\mathrm{H} 4$ (that is, K5, K8, K12 and K16). When compared to whole genome chromatin, nucleosomes bound by all three Brd proteins were enriched with the $\mathrm{H} 4$ diacetylated forms (K5unK8acK12unK16ac, K5unK8acK12acK16un and K5acK8unK12unK16ac), while only Brd3- and Brd4-bound nucleosomes were enriched in the K5acK8unK12acK16un species (Figure 3b, heatmap; Additional file 3). These results suggest that Brds will tolerate $\mathrm{H} 4$ containing $\mathrm{K} 16 \mathrm{ac}$, although it is not required for binding by the Brds. As depicted in Figure 3b, the nucleosomes bound separately by all three Brd family member proteins were also highly enriched with all $\mathrm{H} 4$ triacetylated forms when compared to whole-genome chromatin, such as the H4K5unK8acK12acK16ac (4.4- to 7.2-fold), H4K5acK8unK12acK16ac (4.7to 7.0-fold), H4K5acK8acK12unK16ac (4.3- to 20.3-fold) and especially H4K5acK8acK12acK16un (11.3- to 44.9fold). $t$-Tests performed with data from three independent experiments for each ChIP revealed that these fold changes are indeed significant as the $P$-values were all $<1 \times 10^{-2}$ (Additional file 4). The finding that H4K5acK8acK12acK16un is the most enriched triacetylated $\mathrm{H} 4$ code further supports the hypothesis that H4K16 acetylation is not a Brd binding site. The tetracetylated $\mathrm{H} 4$ code, H4K5acK8acK12acK16ac, is found on $0.5 \%$ of all $\mathrm{H} 4$ in whole genome chromatin in HEK 293 cells; however, this form is strikingly enriched in nucleosomes bound by all three Brds Brd2 (20.8\%), Brd3 (6.8\%) and Brd4 (9.3\%) - and nearly undetectable in HP1-bound nucleosomes. Moreover, HP1 nucleosomes were depleted of most combinatorial forms of $\mathrm{H} 4$ acetylations compared to whole genome chromatin (Figure 3b).

H4K20me2 is the most abundant mark on human histone $\mathrm{H} 4$, found on approximately $58 \%$ of all $\mathrm{H} 4$ in HEK 293 cells (Additional file 2), consistent with its incorporation in heterochromatin as $\geq 70 \%$ of mammalian genomes are thought to be heterochromatic. H4K20me2 was enriched in HP1-bound nucleosomes and depleted in Brd nucleosomes (Figure 3b). H4K20me2 was especially depleted in Brd4-bound nucleosomes (approximately 8.5\% abundance). Although this mark is extremely high in HP1bound nucleosomes, approximately $80 \%$ for HP $1 \beta$ and approximately $73 \%$ for $\mathrm{HP} 1 \alpha$, we lack direct evidence to indicate that the HP1 chromodomains are reading this mark (Additional file 2). Similarly to H4K16ac, H4K20me2 may be part of a more complex heterochromatic histone code recognized by an HP1 complex. Additionally, as HP1 is known to bind $\mathrm{H} 3 \mathrm{~K} 9 \mathrm{me} 3$, it is reasonable to speculate that the genomic location of this heterochromatic mark overlaps with H4K20me2.

\section{Characterization of PTMs on histone $\mathrm{H} 3$ from Brd- and HP1-associated nucleosomes}

As indicated in Figures 2c and 3a, Brd-bound nucleosomes all have elevated levels of H3K4me1, a gene activation mark. HP1-bound nucleosomes had relatively low levels of this mark. Moreover, H3K4me2 and H3K4me3 were also elevated in the Brd-bound nucleosomes and depleted in HP1-bound nucleosomes. When compared to whole genome chromatin, nucleosomes bound by $\operatorname{Brd} 2$ were the most enriched in these modifications; 12.4 -fold $(P$-value $=$ $0.01)$ for H3K4me2 and 23.6-fold $(P$-value $=0.0009)$ for H3K4me3 (Figures 2c and 3a; Additional file 5). These particular PTMs occur on the nucleosomes surrounding the promoters of actively transcribed genes and are at very low levels genome-wide $(\leq 1 \%)$.

The chromodomains of HP1 $\alpha / \beta$ bind directly to silencing marks H3K9me2 and H3K9me3 [12,21]. HP1-bound nucleosomes were enriched with both these $\mathrm{K} 9$ methylations (Figure 2c). For these modifications, a 1.5- to 2-fold 
increase is significant, as these modifications are extremely abundant in whole-genome chromatin, 31\% and $21 \%$, respectively (Additional file 2). We also found that Brd-bound nucleosomes were low in $\mathrm{H} 3 \mathrm{~K} 9 \mathrm{me} 3$, but have moderate levels of H3K9me2 (Figure 2c).

Another modification, H3K23me1, is also found at low abundance $(0.2 \%)$, but was highly enriched on both HP1s (Figure 2c; Additional file 2). This result is not surprising as a recent study employing a histone peptide methyllysine array found that HP1 $\beta$ binds H3K23me1 in addition to H3K9me3 [22] and colocalizes with H3K9me3 and HP1 $\beta$ at heterochromatic loci. We believe that a subset of HP1 proteins may bind H3K23me1 via its chromodomain independent of H3K9me3 within these loci. H3K18me1 is another modification that is of very low relative abundance in whole genome chromatin (0.3\%). Here we find that Brdbound nucleosomes were highly enriched with this modification; Brd2 (5.23\%), Brd3 (4.17\%) and Brd4 (3.10\%) (Additional file 2). H3K18 can also be acetylated by the CBP/p300 acetyltransferase, and H3K18ac localizes with H3K4me3 by ChIP at the promoters of some actively transcribed genes [3,23-25]. As depicted in Figure 2c, nucleosomes bound by Brds were enriched in H3K18ac (4.2- to 6.9-fold; $P$-values $<0.001$; Additional file 5) compared to bulk chromatin. This result is consistent with reports that H3K18ac localizes with H3K4me3, as Brd nucleosomes were also enriched with H3K4me3. The nucleosomes bound by the Brds also exhibited high levels of acetylation at H3K9ac, H3K14ac, and H3K23ac, whereas acetylation of these residues was reduced on HP1-associated nucleosomes (Figure 2c).

Two other abundant gene repressive PTMs are H3K27me3 and H3K27me2. Nucleosomes bound by both HP1 proteins were modestly enriched with these modifications (Figure 2c). H3K27me3 and H3K27me2 are thought to demarcate the promoter regions and bodies, respectively, of particular repressed genes. These methylations are added by the polycomb group proteins Ezh1 and Ezh2 and bound by other polycomb group chromodomain-containing CBX proteins [26,27]. Interestingly, our observation that HP1-bound nucleosomes were enriched with $\mathrm{H} 3 \mathrm{~K} 27 \mathrm{me} 2 / 3$ suggests that there may be some overlap amongst heterochromatin bound by the HP1 and polycomb CBX chromodomain proteins. Future ChIP-seq experiments with the polycomb CBX proteins will be required to determine their genomic overlap with the HP1 proteins.

As some H3 PTMs can be observed on the same peptides, a few combinations of PTMs were also characterized (Additional file 6). We believe that there is useful combinatorial information in tandem PTMs; for example, we previously reported that the histone H3K9 euchromatic dimethytransferase G9a preferentially methylates H3 peptides that contain K14 acetylation [28]. This modification,
H3K9me2K14ac, shares similar characteristics to the 'bivalent domain' (H3K4me3K27me3) found on silent genes poised for activation in pluripotent stem cells, containing PTMs with opposing epigenetic functions [29]. As mentioned previously, Brd-bound nucleosomes contained some H3K9me2, but the bulk of this modification occurred on peptides that were acetylated on the adjacent lysine (H3K9me2K14ac), the tandem marks effected by G9a (Figure 3a). This result is consistent with the observation that G9a localizes primarily to euchromatin, and that H3K9me2K14ac may also act as a combinatorial switch, similar to the bivalent domain. In contrast, H3K9me3 was depleted in Brd nucleosomes even when H3K14 was acetylated. On the other hand, there was substantial amounts of this combinatorial modification, H3K9me3K14ac, on nucleosomes bound by $\mathrm{HP} 1 \alpha(13.7 \%)$ and HP1 $(16.3 \%)$, which occurs on only $9.8 \%$ of the nucleosomes in the genome (Additional file 6).

Brd-bound nucleosomes also contain histone $\mathrm{H} 3$ enriched with other combinatorial PTMs consistent with gene activation, such as H3K9acK14ac (2.9- to 4.6fold; $P$-values $<0.038)$ and H3K18acK23ac $(5.0$ - to 8.2fold; $P$-values $<0.0008$; Additional file 7) and are depleted in marks that correspond with gene repression, such as H3K9me3K14un (Figure 3c). H3K36me3 is another modification associated with the bodies of actively transcribed genes. We found that Brd4-bound nucleosomes were enriched with this modification (2.2fold), but only when the H3K27 was monomethylated (H3K27me1K36me3) (Figures 2c and 3a).

\section{Chromosomal locations of Brd- and HP1-bound nucleosomes (ChIP-Seq)}

To determine where the histone code reading proteins genomically reside, we deep sequenced the DNA isolated in Brd- and HP1-bound nucleosomes (ChIP-seq) [4]. Figure 4a shows Brd and HP1 ChIP-seq maps for chromosomes $17, \times$ and 12 . At this level of resolution there is much overlap of the ChIP-seq maps amongst the three Brds and amongst the two HP1s. In agreement with previous reports we observed a cluster of HP1 binding that mapped within the centromere region on every chromosome [17]. HP1 binding also mapped along the entire length of the $\times$ chromosome, which is consistent with the fact that HEK293 cells are of female origin, possessing an imprinted inactive $\times$ chromosome. In contrast, Brd-binding clusters were found throughout chromosome 17 but there are only 4 predominant HP1binding clusters, as this is a relatively small chromosome that contains many active genes, including the HOXB cluster (HOX gene clusters will be discussed later). Other chromosomes such as chromosome 12 had a more uniform distribution of Brd- and HP1-binding clusters. 
(a)

Brd2
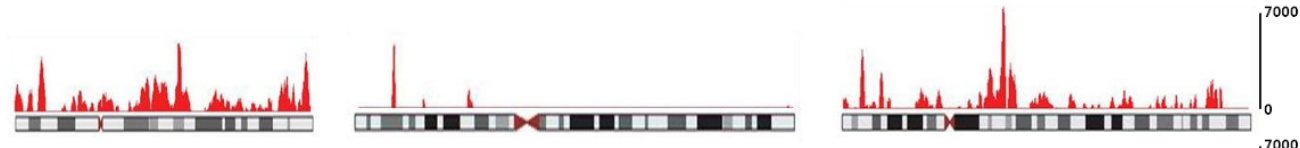

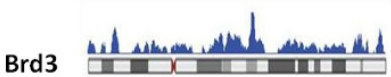
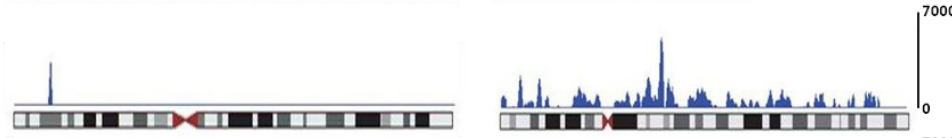

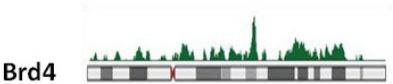
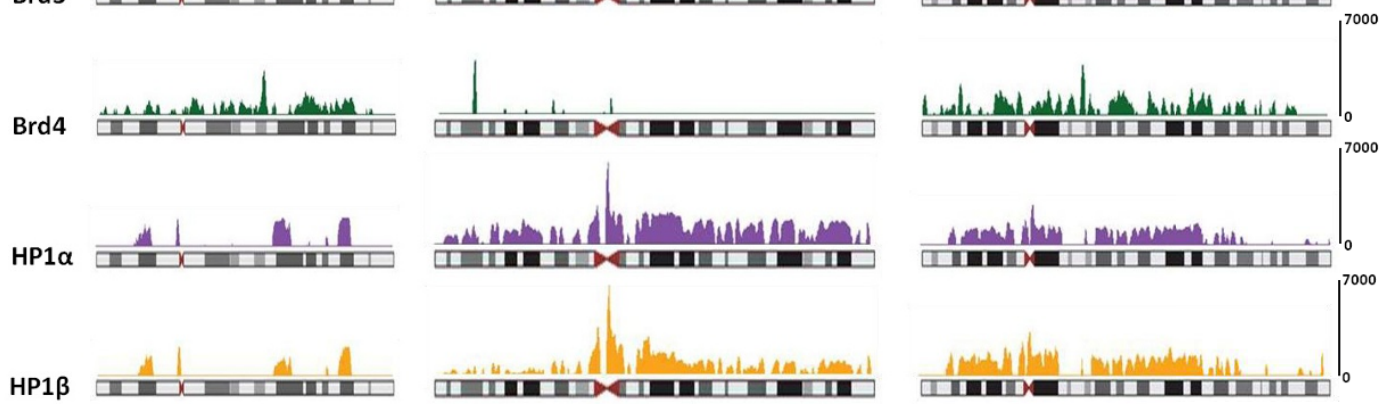

Chromosome 17

X Chromosome

Chromosome 12

(b)

\begin{tabular}{|c|c|c|c|c|c|}
\hline ChIP & Promoter & Gene Body & Intergenic & Aligned Tags & Peaks \\
\hline Brd2 & $13 \%$ & $54 \%$ & $33 \%$ & 11502558 & 48406 \\
\hline Brd3 & $13 \%$ & $52 \%$ & $35 \%$ & 10949188 & 32756 \\
\hline Brd4 & $9 \%$ & $52 \%$ & $39 \%$ & 11051741 & 22427 \\
\hline HP1 $\alpha$ & $2 \%$ & $25 \%$ & $73 \%$ & 7754750 & 1944 \\
\hline HP1 $\beta$ & $1 \%$ & $33 \%$ & $66 \%$ & 8200301 & 10848 \\
\hline
\end{tabular}

(d)

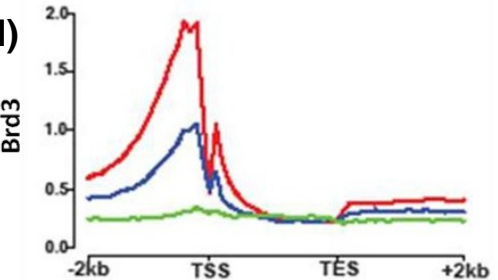

(f)

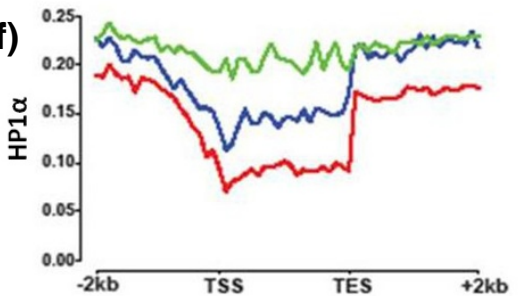

(c)

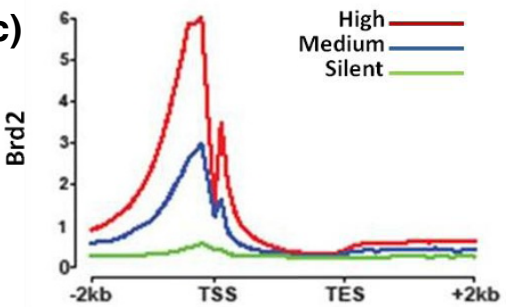

(e)
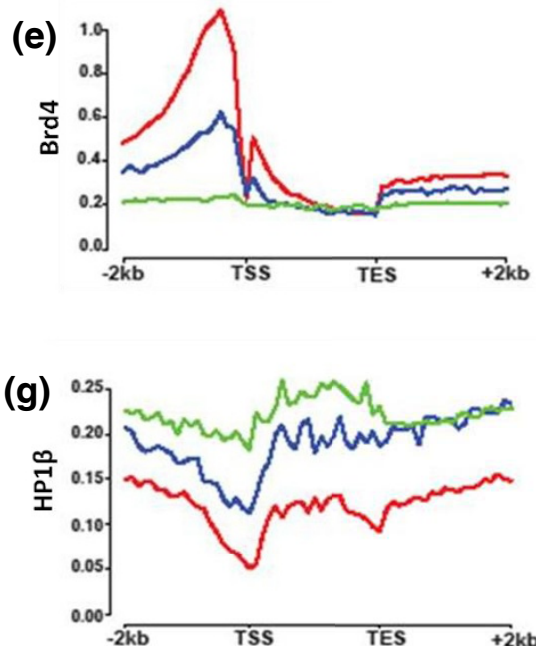

Figure 4 Mapping of Brd- and HP1-bound nucleosomes to their genomic locations. (a) Chromosome maps showing the density of Brdand HP1-bound nucleosomes generated by Solexa Deep sequencing on chromosomes 17, $\times$ and 12. (b) Table denoting the enrichment of Brd and HP1 nucleosomes within the genome relative to the genes. (c-g) Graphs showing the relative enrichment of Brd and HP1 nucleosomes within genes, grouped by expression levels (high, red; medium, blue; silent, green). TES, transcription end site; TSS, transcription start site. 
At the gene level (Figure $4 \mathrm{~b}$ ), approximately $65 \%$ of Brdbinding sites were found on genes (promoter plus gene body). The remaining approximately $35 \%$ was found on intergenic regions, very often in regions flanking genes. The promoter regions as defined in this study are the $+2 \mathrm{~kb}$ regions upstream of the transcription start sites. We observed that 9 to $13 \%$ of the Brd-binding sites were found within such promoter regions. On the other hand, HP1 $\alpha$ and $\beta$ binding sites were predominately found in intergenic regions (73\% and 66\%, respectively) and rarely on promoter regions of genes (1 to $2 \%$ ). Complete lists of promoters bound by Brd and HP1 ChIPs can be found in Additional file 8. As shown in Figure 4c-e, patterns of Brd binding indicate that these proteins are predominately found on high and moderately expressed genes and rarely on transcriptionally silent genes. Genes were ranked based on their expression levels as determined by RNA-Seq, where high expression is defined as the top third of the genes, the middle third as moderate and the bottom third as silent. As predicted, the vast majority of genes bound by the HP1s were classified as either silent or having moderate expression (Figure 4f,g). A heatmap depicting the DNA consensus motifs enriched in the ChIPs can be found in Additional file 9 with an accompanying list of motifs (Additional file 10). We have also included lists of Gene Ontology terms enriched in the Brd ChIPs (Additional file 11).

\section{Brd-bound nucleosomes are associated with the promoters of HOX genes}

To more fully probe the genes bound by each reader, we focused on those genes whose promoters had the most significant binding. As shown in Figure 5a, genes were grouped according to the combinatorial promoter binding patterns of Brd and HP1 proteins (bound genes in red and unbound genes in blue), and expressed and non-expressed genes are shown in red and blue, respectively. We found substantial co-binding of gene promoters by the Brd proteins and a strong correlation between Brd binding and gene expression. Considering the top 500 most Brdenriched gene promoters, we found a $54 \%$ overlap amongst Brd2 and Brd3, a 35\% overlap amongst Brd3 and Brd4, and a $28 \%$ overlap between Brd2 and Brd4. As for particular genes, we observed from an unbiased ranked ordered list of bound promoters (Figure $5 b$ ) that an unusually large number of the highest ranking promoters bound by the Brd proteins were HOX genes. We have also validated the binding of endogenous Brd4 to several HOX genes (HOXB5, HOXB3, HOXC5 and HOXC11) by ChIP, using a monoclonal antibody raised against Brd4 (Additional file 12). There are four HOX gene clusters (HOXA to HOXD) in the human genome, each containing between 10 and 12 protein coding Hox (homeobox) genes as well as several microRNAs [30]. As shown in Figure 5b, when considering the top 35 promoters bound by $\mathrm{Brd} 3$ and Brd4, 18 and 15, respectively, are from HOX gene clusters. When we considered the top 100 scoring gene promoters bound by both $\operatorname{Brd} 3$ and Brd4, we found in both cases they included 15 HOX (homeobox) encoding genes and $3 \mathrm{HOX}$ cluster encoded microRNAs all found within the HOXA, HOXB and HOXC clusters. As for Brd2 promoter binding, we found 9 of the top 100 ranked genes were HOX genes. Although we were surprised to find such a huge enrichment of HOX genes in Brd ChIPs, this result is in agreement with the fact that the Brd proteins are human homologs of the Drosophila FSH-S and FSH-L proteins, which are encoded by trithorax group genes [6]. Trithorax group genes were originally identified from Drosophila genetic screens as genes required for the persistent expression of HOX genes that control the development of body segments [31,32]. The differential expression of HOX genes is also essential for patterning of all vertebrate embryos and the correct sustained expression of HOX genes in different tissue types is required to maintain cells in their respective differentiated states [33]. Therefore, maintaining a specific chromatin architecture at $\mathrm{HOX}$ gene clusters is an elegant evolutionary solution to regulate their expression, potentially through epigenetic regulation. Other trithorax proteins that regulate HOX gene expression have been shown to be components of chromatin remodeling, histone methylation and histone acetylation complexes [34-36]. In fact, the Drosophila trithorax acetylation complex (TAC1) and the Drosophila Brd protein (FSH-S) are required for the expression of the HOX gene $U b x$ and the overexpression of FSH-S can induce the ectopic expression of several HOX genes in Drosophila [6,34]. These results suggest that the histone acetylation marks that are written by $\mathrm{TAC} 1$ are read and translated by the Brd protein FSH-S.

\section{HP1 bound nucleosomes are associated with the promoters of ZNF genes on chromosome 19}

As stated above, the HP1s were found at the promoter regions of 1 to $2 \%$ of the genes they bind and most of these are transcriptionally silent (Figure $4 \mathrm{~b}, \mathrm{f}, \mathrm{g}$ ). Moreover, neither HP1 protein was found on any HOX gene promoters. We also did not observe an equal distribution amongst the chromosomes with genes whose promoters were bound by the HP1 proteins. For both HP1 proteins, approximately $30 \%$ were found on chromosome 19 and $20 \%$ were found on the $\times$ chromosome. As shown in Figure $5 \mathrm{~b}$, when we only considered the top 35 gene promoters bound by HP1 proteins, HP1 $\alpha$ and HP1 $\beta$ bound 18 and 15 ZNF gene promoters, respectively, from chromosome 19. ZNF genes encode proteins that belong to a large superfamily of putative transcription factors (approximately 800 members in humans), many of which are thought to function as transcriptional repressors [37]. 
(a)

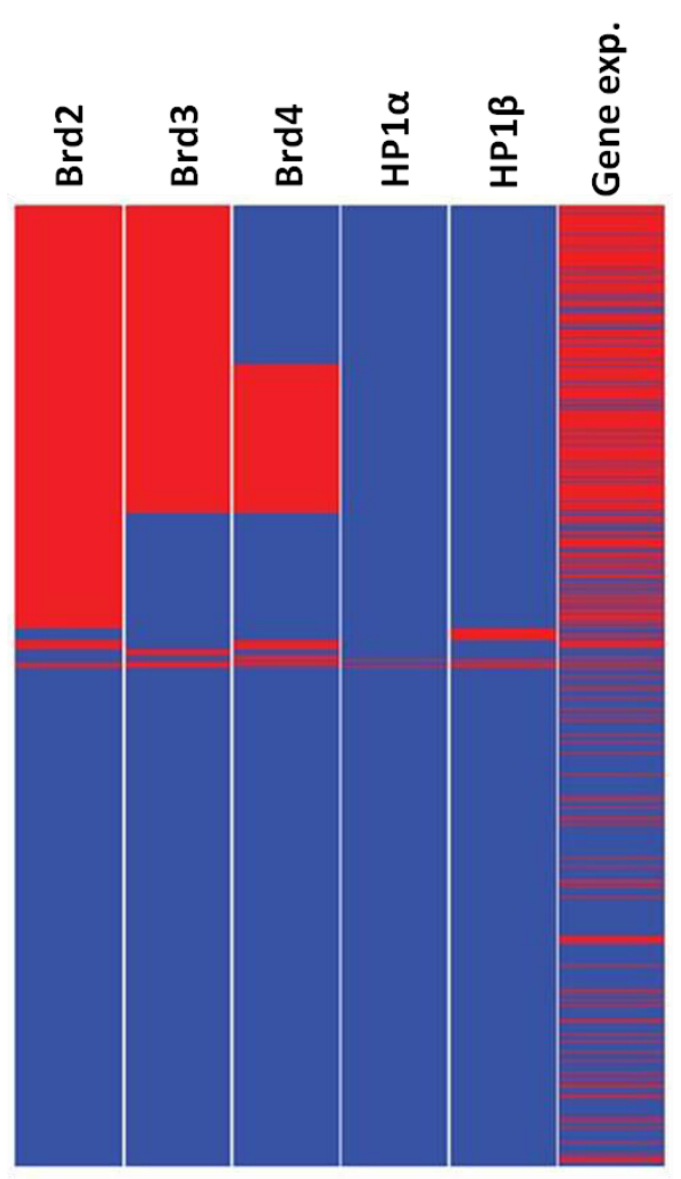

(b)

\begin{tabular}{|c|c|c|c|c|}
\hline BRD2 & BRD3 & BRD4 & HP1 $\alpha$ & HP1 $\beta$ \\
\hline CYR61 & hsa-mir-10a & hsa-mir-10a & AC078778.34 & RP11-3D23.1 \\
\hline HOXA11 & HOXB3 & HOXB3 & IGHV1OR15-9 & AL732371.4 \\
\hline AC084018.28 & HOXB4 & HOXB5 & AP001005.6 & AL365179.30 \\
\hline SNRPA & hsa-mir-196a-2 & HOXB4 & RP11-3D23.1 & AC012309.8 \\
\hline SNORD58 & HOXC9 & ZNF608 & AC134882.2 & IGHV1OR15-9 \\
\hline RNF5 & HOXC8 & ALPK2 & ZNF233 & ZNF404 \\
\hline H3F3B & HOXC10 & AC104971.5 & ANKRD36 & ZNF233 \\
\hline HOXD11 & SMAD7 & NR2F2 & AL732363.8 & AP001005.6 \\
\hline RXRB & HOXA6 & HOXB2 & AC134882.2 & AL845433.4 \\
\hline FASTK & HOXA7 & HOXA4 & AC012309.8 & AC069278.1 \\
\hline Y_RNA & HOXA5 & LSM14A & RP11-764K9.4 & AC134882.2 \\
\hline AGAP3 & HOXA3 & PRKCBP1 & FANK1 & AC022148.6 \\
\hline ZNF503 & TFAP2A & HOXB7 & ZNF221 & ZNF793 \\
\hline hsa-mir-93 & PSG5 & HOXB8 & AC012309.8 & ZNF573 \\
\hline NQO2 & CAPN2 & HOXB6 & 5S_rRNA & AC016582.9 \\
\hline IER2 & SMUG1 & TFAP2A & ZNF404 & ZNF30 \\
\hline RNF167 & AC103702.3 & AL033381.2 & ZNF284 & ZNF222 \\
\hline C18orf32 & HOXB6 & AC008080.1 & AC098691.2 & C19orf18 \\
\hline BCL11A & HOXB5 & HOXC5 & 5S_rRNA & VN1R7P \\
\hline SNORD58 & STRA6 & hsa-mir-615 & AL512625.10 & EIF3S5P \\
\hline PPT2 & HOXC5 & RNF43 & ZNF226 & AC022148.6 \\
\hline HOXA1 & hsa-mir-615 & MYO10 & ZNF781 & AC092295.2 \\
\hline SOX15 & AC008080.1 & LIN28B & AC093227.3 & ZNF585A \\
\hline $\mathrm{ZC} 3 \mathrm{H} 4$ & PRKCBP1 & MARCKS & 5_8S_rRNA & RP1-278E11.5 \\
\hline PFN1 & NR1D1 & CAPN2 & SSU_rRNA_5 & AL136089.15 \\
\hline AC100778.5 & AC068669.21 & AL662890.3 & ZNF225 & CEACAMP11 \\
\hline snoR38 & SERTAD3 & HOXC11 & EIF3S5P & ZNF225 \\
\hline ZNF507 & SERTAD1 & SEC22B & ZNF471 & AL662795.5 \\
\hline NRP1 & AL662890.3 & TRDMT1 & RP1-278E11.5 & ZNF419 \\
\hline FAM83B & HOXC6 & RP11-30A20.3 & ZNF30 & AC008507.11 \\
\hline DDX11 & HOXC4 & HOXA5 & CR848007.2 & ZNF226 \\
\hline BHLHB2 & ACTN4 & HOXA11 & HLA-DQB1 & ZNF134 \\
\hline PCDH7 & HOXB2 & HOXA10 & AC069278.1 & ZNF211 \\
\hline SFMBT2 & BCL6B & SERTAD3 & Y_RNA & AC026786.5 \\
\hline SNORD58 & C17orf49 & SERTAD1 & ZNF302 & ZNF781 \\
\hline \multicolumn{2}{|c|}{ Cluster } & \multicolumn{3}{|c|}{ Chromosome } \\
\hline \multicolumn{2}{|c|}{ HOXA } & \multicolumn{3}{|c|}{7} \\
\hline \multicolumn{2}{|c|}{ HOXB } & \multicolumn{3}{|c|}{17} \\
\hline \multirow{2}{*}{\multicolumn{2}{|c|}{ HOXC }} & \multicolumn{3}{|c|}{12} \\
\hline & & \multicolumn{3}{|c|}{2} \\
\hline \multicolumn{2}{|c|}{ ZNF } & & 19 & \\
\hline
\end{tabular}

3

(c)
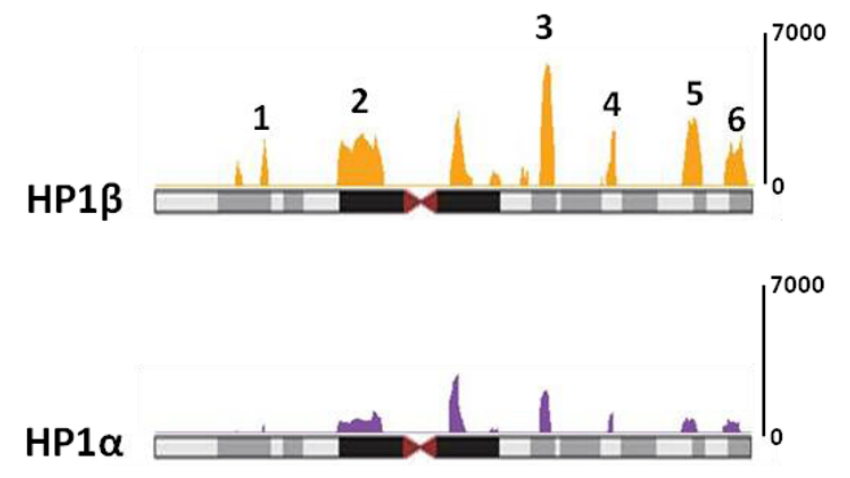

\section{Chromosome 19}

Figure 5 Promoters bound by Brd- and HP1-bound nucleosomes. (a) Heatmap of promoters with nucleosomes bound by the Brd and HP1 proteins. Heatmaps are arranged as clusters of promoters bound by the various ChIPs (lanes 1 to 5: bound, red; unbound, blue) and the expression of these genes from RNA-Seq is represented in lane 6 (high and medium, red; silent, blue). (b) Ranked order table of genes (top 35) whose promoters are bound by the Brd and HP1 proteins. Genes are ordered based on Brd/HP1 binding score within the promoter region of all annotated genes (top of the list, most bound). Genes belonging to the HOX clusters or ZNF clusters are color coded accordingly. (c) HP1 $\alpha$ and HP1 $\beta$ binding clusters on chromosome 19. The ZNF clusters are labeled 1 to 6. 
There are six clusters of ZNF genes found on chromosome 19. These clusters all span from 1 to $3 \mathrm{Mb}$ of DNA each and are located along chromosome 19 as follows: cluster 1, 10 to $12 \mathrm{Mb}$; cluster 2, 19 to $23 \mathrm{Mb}$; cluster 3, 40 to $43 \mathrm{Mb}$; cluster 4,49 to $50 \mathrm{Mb}$; cluster 5,57 to 59 $\mathrm{Mb}$; and cluster 6, 61 to $63 \mathrm{Mb}$ (Figure $5 \mathrm{c}$ ). When considering the top 100 bound promoters, we observed that HP1 $\alpha$ bound to 37 gene promoters ( 23 known ZNF genes), and HP1 $\beta$ to 55 gene promoters (27 known ZNF genes), all located within these clusters on chromosome 19. However, we did not see complete overlap amongst the promoter binding of both HP1 proteins. While we found extensive binding of HP1 $1 \beta$ to the promoters of genes in all six clusters, HP1 $\alpha$ was only found predominantly in clusters 3,4 and 6 , with very little binding in the other three clusters. Interestingly, the promoters of genes found in cluster 5 are extensively bound by HP $1 \beta$ and almost completely devoid of HP1 $\alpha$ binding. Within this cluster, HP1 $\beta$ was bound to the promoters of 59 genes, 16 of which are ZNF genes, as well as a subcluster of 20 microRNA genes in the distal half of this cluster. These data are consistent with a study in 2006 that used a DNA adenine methyltransferase identification (DamID) method coupled with cDNA oligo microarrays to identify the target genes bound by HP1 $\beta$ [38]. In agreement with these findings, ChIP-seq experiments with antibodies directed against H3K9me3 have found distinct islands of this modification map to the ZNF clusters [4].

\section{Brd4 is a positive regulator of the genes it binds}

In order to determine whether Brds directly regulate the expression of the genes they bind, we performed gene expression microarrays on cells depleted of Brd4 by short hairpin RNA (shRNA). We choose to knockdown Brd4 because it shared the least overall overlap of bound promoters with the other Brd ChIPs. We achieved a threefold knockdown of Brd4 with a custom shRNA in HEK293 cells as determined by RT-PCR. The knockdown was also verified at the protein level by western blot (Additional file 13). Of the top 100 genes whose promoters were most bound by Brd4, we found three-quarters of them had at least a two-fold reduction in expression in the Brd4 shRNA cell line when compared to the control (Figure 6a, right heatmap). These results suggest that the direct binding of Brd4 to the acetylated nucleosomes associated with these genes is required for their proper expression. Only 8 of these top 100 genes showed an increase in expression upon Brd4 depletion. We speculate that these may be the result of downstream effects rather than a loss of Brd4 binding. Looking at the $28 \mathrm{HOX}$ genes whose promoters were significantly bound by Brd4, we found that twothirds of this subset of genes had reduced expression (1.5fold or greater; Figure $6 \mathrm{~b}$ ). Only 6 of the 28 protein coding HOX genes had an increase in expression and 5 showed no significant change upon Brd4 knockdown. This result suggests that there is redundancy amongst the Brd proteins, as we found overlapping binding of the Brds on many of the HOX genes, especially between $\mathrm{Brd} 3$ and Brd4.

\section{HP1 $\beta$ is a negative regulator of the genes it binds}

In order to determine the direct effects of HP1 binding to nucleosomes, we preformed gene expression arrays on HEK293 cells depleted of HP1 $\beta$ by shRNA. We chose to knockdown HP1 $\beta$ rather than HP1 $\alpha$ because it was enriched on more promoters, including most of the HP1 $\alpha$-bound promoters. Our custom shRNA knocked down HP1 $\beta$ mRNA by 3.5-fold in HEK293 cells as determined by RT-PCR. The knockdown was also verified at the protein level by western blot (Additional file 13). In contrast to the effects of Brd knockdown, knockdown of HP $1 \beta$ resulted in at least two-fold increases expression in two-thirds of the top 100 HP1 $\beta$ promoter-bound genes (Figure 6a, left heatmap). These results suggest that the binding of HP1 $\beta$ to histone methylated nucleosomes is required for effective silencing of associated genes. We also looked at the expression of the ZNF genes found on chromosome 19 whose promoters were enriched with HP1 $\beta$-bound nucleosomes (Figure 6b, left heatmap). Surprisingly, we found that the expression of only one-third of these genes significantly increased, whereas two-thirds remained the same or decreased. This result is in agreement with the aforementioned study that mapped HP $1 \beta$ to the ZNF clusters on chromosome 19 with the DamID method [38]. They also reported that individual depletion of $\mathrm{HP} 1 \alpha / \beta$ did not lead to an appreciable change in the expression of most of the ZNF clusters. We hypothesize that this is due to the redundancy of the three HP1s (HP1 $\alpha, \beta$ and $\gamma$ ) and attempts to circumvent this by depleting more than one at a time have been unsuccessful. Also, removing the heterochromatic binding proteins alone may not be enough to fully turn on repressed genes, as there are other steps required for gene activation, such as recruitment of transcription factors or gene activating histone-modifying enzymes or other binding proteins. This hypothesis would also be consistent with our finding that the expression of the majority of genes whose promoter regions were bound by HP1 $\beta$ did not change significantly when HP1 $\beta$ was depleted.

\section{Brd-bound nucleosomes are associated throughout the body of transcribed HOX genes}

Having selected for significantly bound genes based on Brd and HP1 promoter binding, we next looked at the binding of these proteins across the entire length of such genes. For actively transcribed genes, we examined Brd and HP1 binding across the HOXA10, HOXA11, HOXB9, and HOXC10 genes and the housekeeping gene 


\section{(a) Brd4 KD}

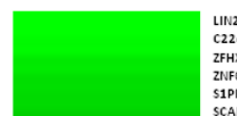

\section{HP1ß KD}

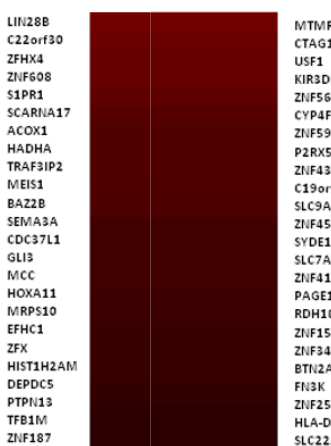

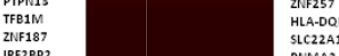

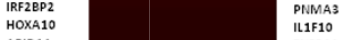

ARIO1A
HOXE2

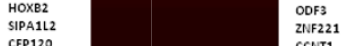

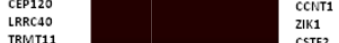

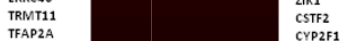

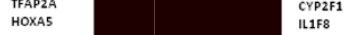

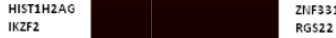

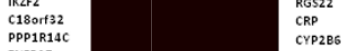

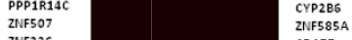

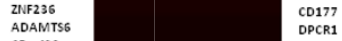

C5ort39
C6ort26

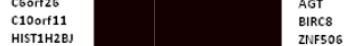

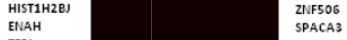

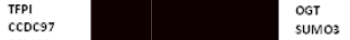

ReMS3
ROXA7

LGALS13
HOXXE
CLECGM

MLTS
CETM3
MOSH

CETN3
GNPAT

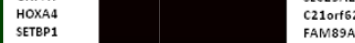

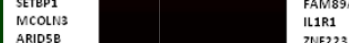

ARIOSB
CHCHD1
CHAFF23
IIIF5

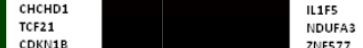

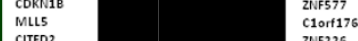

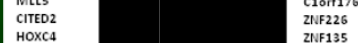

Hoxc4
Hoxed
Texe

ZEE2
SERTAD1
ZNFA1

SERTAO1
STI1
C10r55

HNMT
OAYOSO

\begin{tabular}{l|l} 
FoXp2 \\
NiR2F2 \\
NR2
\end{tabular}

HADHB

LSM14A

SRPP

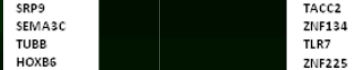

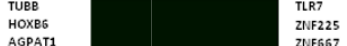

соMT

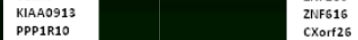

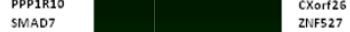

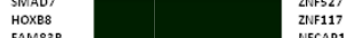

FAMB38
HOXE3

\begin{tabular}{l|l} 
SLC1A3 \\
SEC228 \\
ZNEF573 \\
TEX11
\end{tabular}

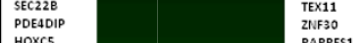

HOXCS
MRPS18B
MASB

MRPS18B
MAT2E

\begin{tabular}{l|l} 
HoXAG \\
JUP
\end{tabular}

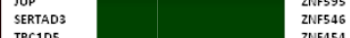

TRC105
STRAG
TRAT

NuAK1
ALPK2

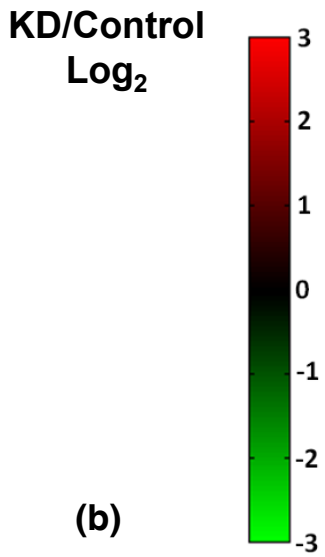

HP1ß KD

Brd4 KD

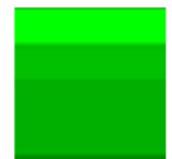

HOXC8

HOXA13

HOXA11

HOXA11AS

HOXA10

HOXA9

HOXB2

HOXA5

HOXC10

HOXA7

HOXD11

HOXB5

HOXB9

HOXA4

HOXC4

HOXB4

HOXC9

HOXB6

HOXB8

HOXB3

HOXD12

HOXC5

HOXAG

HOXB1

HOXA2

HOXC11

HOXB7

HOXC6

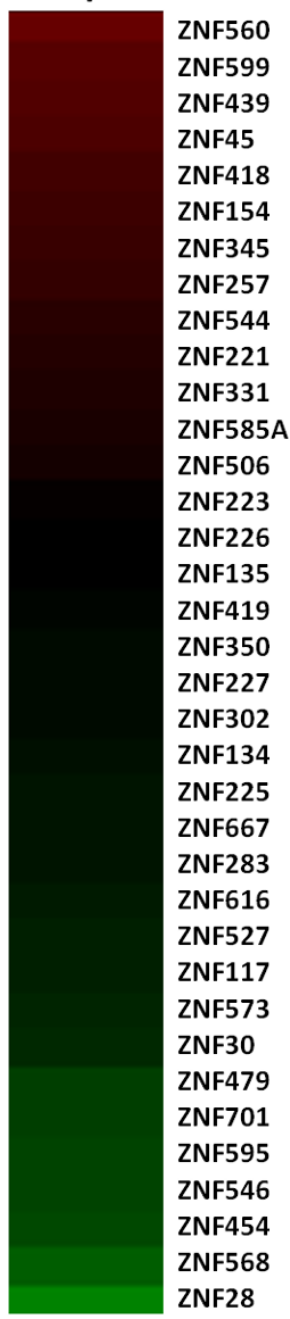

Figure 6 Brd4 and HP1 $\beta$ regulate gene expression. (a) Right panel: heatmap showing the $\log _{2}$ fold change in gene expression from 293 cells with depleted Brd4 compared to control 293 cells. Genes shown are the top 100 genes whose promoter regions were the most enriched with Brd4 bound nucleosomes. Left panel: heatmap showing the $\log _{2}$ fold change in gene expression from 293 cells with depleted HP1 $\beta$ compared to control 293 cells. Genes shown are the top 100 genes whose promoter regions were the most enriched with HP1 $\beta$-bound nucleosomes. (b) Right panel: heatmap of the $\log _{2}$ fold change in expression of all HOX genes with promoters bound by Brd4 in the Brd4depleted 293 cell line compared to control 293 cells. Left panel: heatmap of the $\log _{2}$ fold change in expression of all ZNF genes found on chromosome 19 with promoters bound by HP1 13 in the HP1 $\beta$-depleted 293 cell line compared to control 293 cells. 
$A C T B$ (Figure $7 \mathrm{a}-\mathrm{d}$ ), and these genes were found to be devoid of HP1. We also found that the expression of these genes were all significantly reduced in the Brd4 knockdown cells: HOXA10 by 3 -fold, HOXA11 by 4fold, HOXB9 by 2 -fold, HOXC10 by 2.5 -fold and ACTB by 1.5 -fold. The mRNA for the housekeeping gene $A C T B$ is extremely abundant, so we believe that a 1.5fold decrease in its expression is significant. Moreover, the expression of this gene increased by 1.4-fold in the HP1 13 kockdown cells. As shown in Figure 7a,c, binding of all three Brds was found to be evenly distributed throughout the promoter region and gene bodies of the HOXA10, HOXA11 and HOXC10 genes. On the HOXB9 gene we found a huge enrichment of $\mathrm{Brd} 2$ and $\mathrm{Brd} 3$ within the promoter region (Figure $7 \mathrm{~b}$ ). Both proteins were also bound to the body of this gene with Brd2 enrichment peaking in the later half towards the 3' region. Brd4 binding to $H O X B 9$ nucleosomes was evenly distributed over the promoter region and gene body. As for the housekeeping $A C T B$ gene, we found that all three Brd proteins bound within the promoter region and the gene body with Brd2 being especially enriched with the body of this gene. This is consistent with the role of Brd proteins in transcriptional elongation $[8,39]$. Furthermore, the binding of Brd proteins to the bodies of HOX genes is also consistent with the reported hyperacetylation of histone $\mathrm{H} 4$ within the bodies of HOXA genes upon activation with retinoic acid in pluripotent embryonal carcinoma cells [40].

\section{HP1-bound nucleosomes are associated with the bodies of transcriptionally silent ZNF genes}

We next analyzed the binding patterns of HP1 proteins on the ZNF560 and ZNF599 genes (Figure 7e,f). Both genes were determined to be silent in control HEK293 cells by RNA-Seq analysis (Figure 7e,f, bottom panels). ZNF560 and ZNF599 are found in the HP1-binding clusters 3 and 6 on chromosome 19, which are significantly bound by both HP1 $\alpha$ and HP1 $\beta$. We chose these ZNF genes for further analysis as their expression was the most upregulated of all HP1-bound ZNF genes upon depletion of HP $1 \beta$ by shRNA (Figure 6b). As depicted in Figure 7e,f, both HP1s bound nucleosomes throughout the bodies and promoter regions of ZNF599 and ZNF560, with HP1 $\beta$ being slightly more enriched within the promoter and 5 , region of ZNF599. These results, along with our array analysis, suggest that the HP1 proteins directly repress the expression of these genes. Moreover, nucleosomes found within both of these genes are relatively devoid of binding by all three Brds. Interestingly, we did observe a short cluster of Brd-bound nucleosomes at the end of the ZNF599 gene and continuing in the 3' direction on chromosome 19; however, this result is rather perplexing as there are no actively transcribed genes in this region.

\section{Discussion}

Since the completion of the Human Genome Project in 2000 , there have been several calls for a similar project to catalog human epigenomes. In 2008 the Alliance for the Human Epigenome and Disease (AHEAD) outlined the need for a project to provide high-resolution epigenome maps and suggested guidelines for the first pass of a human epigenome reference [41]. At the heart of these initiatives is the use of ChIPs with antibodies that recognize specific PTMs. Such studies are informative; however, ChIPs with modification-specific antibodies will only provide epigenomic maps with little knowledge on how such information is translated. Additionally, site-specific antibodies often suffer from cross-reactivity and epitope occlusion. Our less biased proteomic-genomic approaches complement such ongoing studies and shed light on how histone PTMs are translated into transcriptional outcomes.

Two different models have been proposed for the function of Brd proteins in transcription. In one model Brd4 recruits the transcription elongation factor $\mathrm{pTefb}$ to the transcription complex at the transcription start site, which then phosphorylates the CTD of RNA polymerase II, leading to more processive transcript elongation [39]. In the second model, Brd proteins are recruited to acetylated nucleosomes via their bromodomains and facilitate the passage of RNA polymerase II through nucleosomes by their intrinsic chaperone activity [8]. Our ChIP-seq results support both models as we have observed an enrichment of the Brd proteins at both the promoter (approximately 12\%) and body (approximately 52\%) of active genes. These results suggest an equal distribution of Brd proteins on promoter regions and gene bodies, as we have defined promoters regions as $2 \mathrm{~kb}$ upstream of the transcription start site and the average gene body is 10 to $20 \mathrm{~kb}$. Moreover, the Brd-bound nucleosomes were enriched with patterns of histone PTMs found in both the promoter region and body of actively transcribed genes. ChIP-seq experiments with specific antibodies have found that the PTMs H3K9ac and H3K4me3 are associated with only a few nucleosomes at active gene promoters, whereas H3K79me1 and H4K20me1 are exclusively associated with the bodies of active genes $[3,4]$. When compared to whole-genome chromatin, the Brd-bound nucleosomes were enriched in all of these modifications, further suggesting that they represent a mixture of promoter and gene body nucleosomes. The Brd-bound nucleosomes were also enriched with the histone PTMs H3K14ac (approximately 55\%), H3K23ac (approximately 50\%), H4K12ac (approximately 50\%) and H4K16ac (approximately 55\%). These modifications were previously shown to be enriched on both the promoters and bodies of active genes [3,4]. Our data suggest that Brd nucleosomes are a mixture of the following: 


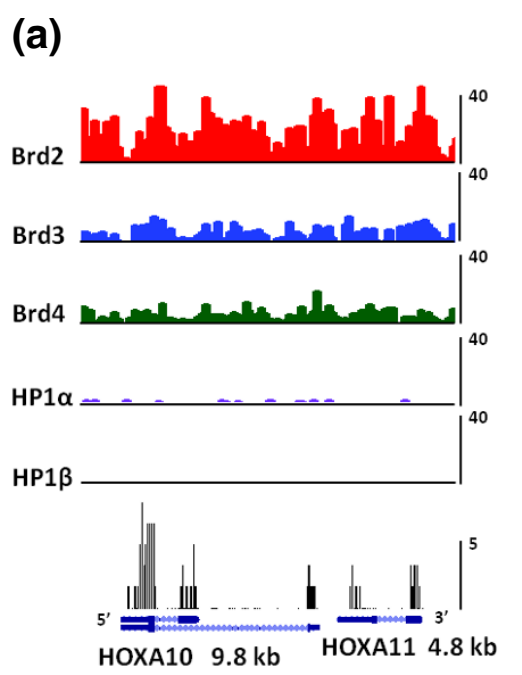

(b)
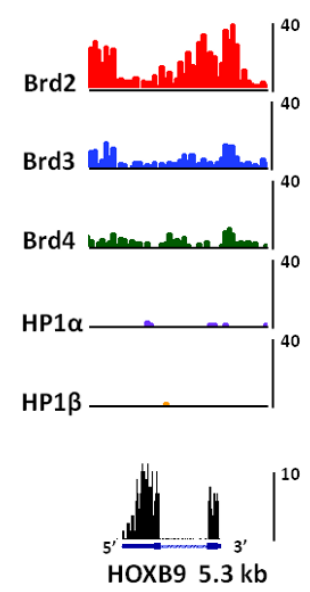

(c)
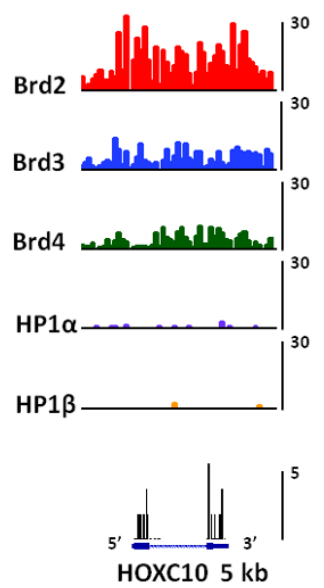

(d)
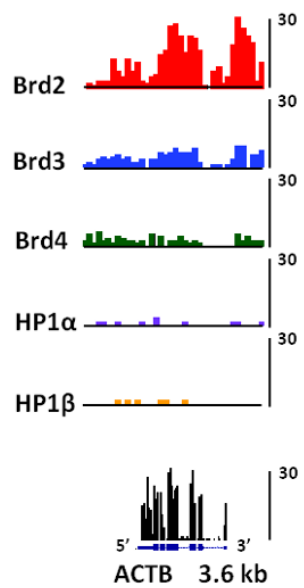

(e)
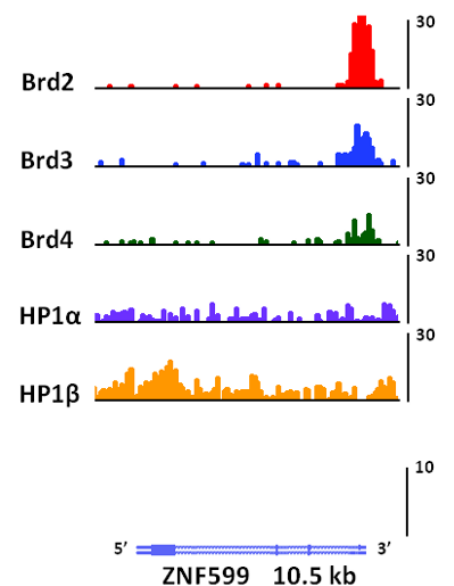

(f)
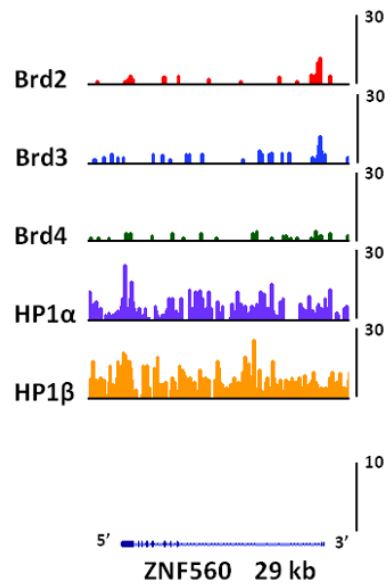

Figure $7 \mathrm{Brd}$ and HP1 protein nucleosomal binding patterns of Brd4 and HP1 $\beta$ regulated genes. (a) HOXA10 and HOXA11. (b) HOXB9. (c) HOXC10. (d) ACTB. (e) ZNF599. (f) ZNF560. Top panels for are the ChIP-seq results depicting the Brd and HP1 binding patterns. Bottom panel is the RNA-Seq results representing the expression level of each gene.

promoter bound with the predominant histone PTM patterns H3(K4me3, K9ac, K14ac, K23ac) and H4(K5ac, K12ac, K16ac), and gene body enriched with H3(K4me1, K14ac, K23ac, K79me1) and H4(K8ac, K12ac, K16ac, K20me1).

HP1-bound nucleosomes were most enriched with histones containing the repressive PTMs, H3K9me2/3 (approximately $85 \%$ combined) and H4K20me $2 / 3$ (approximately $75 \%$ combined). In addition to binding the ZNF clusters of chromosome 19, our ChIP-seq results have revealed that the HP1 proteins reside on centromeric heterochromatin and inactive $\times$ chromatin, both of which harbor these marks $[3,4,17]$. Also in line with our results, previous ChIP-seq experiments have colocalized these modifications to the ZNF repeat clusters on chromosome 19 [4].

Silent Hox genes are a defining hallmark of pluripotent stem cells and their expression is tightly regulated during differentiation [42]. The differential expression patterns of the Hox genes are the master regulators that direct cellular differentiation and maintain particular cell phenotypes [33]. In fact, all of the protein coding Hox genes 
contained bivalent domains, suggesting that they are poised for activation [29]. Moreover, another ChIP-seq study found that nearly half of all bivalent domains were bound by the polycomb PRC1 complex, which contains a chromodomain protein that binds directly to H3K27me3 [43]. Such bivalent domains were defined as containing overlapping PTMs, H3K4me3 and H3K27me3. It is yet unknown what activating or repressive marks on histone $\mathrm{H} 4$ are found within these bivalent domains. Future experiments will be necessary to investigate whether $\mathrm{H} 4$ acetylations that are bound by the Brd proteins are also present in bivalent domains. If so, it will be interesting to investigate whether Brd proteins are also bound to bivalent domain chromatin concurrent with the PRC1 binding or whether PRC1 complex is released prior to $\mathrm{H} 4$ acetylation and Brd binding upon gene activation. In conclusion, the proteomic and genomic techniques presented here can be used to probe the local chromatin environment bound by any chromatin-associated protein. In particular, we have examined the chromatin from genomic regions where bromodomain- or chromodomain-containing proteins are found, and quantified the histone PTMs present. These data can be used to generate hypotheses on how chromatin-associated proteins (or histone code readers) could influence the transcription of specific genes, hence yielding glimpses into how histone codes could be translated. Such experimental approaches could also be applied to any of the histone code readers in systems of biomedical importance, such as during stem cell differentiation or the metastatic progression of cancers.

\section{Materials and methods Chromatin immunoprecipitation}

Mononucleosomes were purified after (micrococcal nuclease) Mnase digestion with FLAG-Brd or FLAG-HP1 proteins as described in [8] from HEK293 cells. FLAGHP1 vectors were described in [44]. All proteins were generated by transient expression as previously described $[8,44]$, and expression assessed by western blots as shown in Additional file 1. The quality of the ChIPs was analyzed by $17 \%$ SDS-PAGE and Coommassie blue staining. The histone protein ChIP inputs and elutions were propionyl derivatized with either 'light' $d_{0^{-}}$or 'heavy' $d_{10^{-}}$ propionic anhydride, trypsinized and prepared for mass spectrometry as previously described in [18] with minor adjustments. 2-Propanol was substituted for methanol during the derivatization reaction as we have found that methanol can modify peptides at low $\mathrm{pH}$.

\section{Liquid chromatography-mass spectrometry-based proteomics}

Histone peptides were separated by reverse phase nanospray liquid chromatography on $\mathrm{C} 18$ resin with an
Agilent 1200 series HPLC. Mass spectrometry was performed on a LTQ-Orbitrap mass spectrometer (ThermoFisher Scientific Inc. Waltham, MA, USA as previously described [18].

\section{Mass spectrometry data analysis}

The LC-MS/MS data sets were analyzed using in-house software. Briefly, the characterization of the histone PTMs was performed using an optimization-based algorithmic framework that simultaneously utilizes mass spectrometry, MS/MS and chromatographic information to identify and quantify the PTMs on the histone peptides. The method automatically considers multiple charge states of all modified peptides and is able to resolve mixed tandem mass spectra resulting from coeluting and isobaric modified peptides using a superposition method, as previously described [45]. The statistical significance of the relative abundance of histone modifications in various ChIPs as compared to the relative abundance of genomic histone PTMs was calculated using a two-tailed, unpaired Student's $t$-test, and the associated $P$-values were generated using Microsoft Excel. Mass spectrometric data were converted from RAW format into mzXML format using ReAdW. The mzXML files were deposited into the Tranche repository and are available for download from the Proteome Commons [46].

\section{Preparation of DNA for deep sequencing analysis}

Nucleosomal DNAs were treated with alkaline phosphatase to remove the 3' phosphates left after MNase digestion and purified by phenol extraction. The DNA fragments (approximately $1 \mu \mathrm{g}$ ) were further prepared using the Solexa ${ }^{\circledR}$ Illumina library kit directly following the manufacturer's protocol. The purified DNA was used directly for cluster generation and sequencing using a Solexa 1G Genome Analyzer (Illumina).

\section{Preparation of RNA for RNA-Seq analysis}

The raw sequence data for the RNA-Seq in HEK293 cells were obtained from [47] (Gene Expression Omnibus sample ID GSM301568). The RNA preparation and sequencing protocols for this RNA-Seq data can be found in [47].

\section{Deep sequencing analysis}

The raw sequence reads were aligned to the hg18 human genome using the Bowtie short read aligner [48]. To avoid possible PCR artifacts, we kept at most one uniquely aligned read at each genomic location per strand. To determine the number of ChIP-Seq sequencing reads in a genomic interval, we shifted the locations of forward and reverse strand tags by $75 \mathrm{bp}$, half the size of the nucleosomal DNA. 
For the chromosomal binding patterns of Brd and HP1 proteins (Figures $4 \mathrm{a}$ and $5 \mathrm{c}$ ), reads were counted in sliding windows of $1 \mathrm{Mb}$ with a step size of $100 \mathrm{~kb}$. The read counts were normalized to the sequencing depth of 5 million non-redundant reads. The signal level in each $1-\mathrm{Mb}$ window was computed as the difference between the normalized read counts in the window in the sample and the control libraries $\left(\mathrm{N}_{\text {sample }}-\mathrm{N}_{\text {control }}\right)$ with the negative values set to zero. A clustering approach described in [49] was used to identify Brd and HP1 protein-enriched domains ('islands') in the human genome from the ChIPSeq data. The percentage overlap (Figure $4 \mathrm{~b}$ ) of the Brd and HP1 islands with the promoter, the gene-body and the inter-genic regions were determined using the gene annotations from the Ensembl database.

The sequence read density (per 100-bp window and 5 million non-redundant reads) was determined along the gene body and in the upstream/downstream 2-kb regions in the sample and control libraries. The signal level in each window was computed as the difference between the read densities in the sample and control libraries, with the negative values set to zero. Genes were ranked based on their expression levels estimated as reads per kilobase per million mapped reads (RPKM [50] and stratified into three expression groups: the 'silent' group (the bottom $30 \%$ genes in the ranked list), the 'high' group (the top 30\% genes), and the 'medium' group (the remaining genes). The mean signal levels over all genes in each group were determined and are displayed as gene-body plots in Figure 4c-g.

The patterns of the combinatorial binding of Brd and HP1 proteins to the gene promoters and their correlation to the gene expression levels were determined as follows. A gene promoter, defined as a $2-\mathrm{kb}$ region upstream of the transcription start site, is deemed bound by the protein if the island (determined as described above) overlaps the promoter. Genes with a particular combinatorial promoter-binding pattern of the five proteins (such as 'red-red-red-blue-blue' in Figure 5a) were grouped. The groups were then ranked based on the number of their member genes and displayed in the descending order in Figure 5a (except for the most populous group, 'blueblue-blue-blue-blue', displayed at the bottom). The expression status of each gene is indicated as expressed ('red') if its RPKM value is at least 1 and not expressed ('blue') otherwise. The UCSC genome browser [51] bedGraph files displayed in Figure $7 \mathrm{a}-\mathrm{f}$ were generated as follows. For the ChIP-Seq data, the 75-bp-shifted nonredundant reads were counted in 200-bp tiling windows along the genome and the read counts were normalized to a library size of 5 million non-redundant uniquely mapped reads in each library. For the RNA-Seq data, the centers of the reads were counted in 20-bp tiling windows along the human genome. We deposited raw and analyzed ChIP-Seq and microarray data in the Gene Expression Omnibus [52].

\section{Brd4 and HP1 $\beta$ knockdown cells}

HEK293 cells were infected with lentiviral MISSION ${ }^{\circledR}$ (Sigma-Aldrich Corporation, St. Louis, MO, USA) shRNA vectors (pLKO.1-puro) harboring shRNAs to Brd4 (clone TRCN0000062223), HP1 $\beta$ (clone TRCN0000021427) or control (empty pLKO.1-puro vector). After 72 hours the infected cells were selected with puromycin according to the manufacturer's protocols (Sigma Aldrich). Knockdowns of target RNAs were analyzed by qRT-PCR. For qRT-PCR, RNA from cells was purified with an RNeasy kit (Qiagen) and prepared with a cDNA synthesis kit (Invitrogen Carlsbad, CA, USA). qRT-PCR reactions were performed with SYBR FAST qPCR supermix (KAPA Biosystems Woburn, MA, USA).

\section{Microarray analysis}

RNA was extracted and purified with an RNeasy kit (Qiagen) from Brd4, HP1 $\beta$ and control shRNA knockdown HEK293 cells. For each sample 400 ng RNA was linearly amplified and labeled with Cy5-CTP using Low RNA Linear Amplification reagents (Agilent Technologies Santa Clara, CA, USA). Reference RNA from wild-type HEK293 cells was purified, amplified and labeled with $\mathrm{Cy} 3$ in the same manner. Equal quantities of the Cy5and Cy3-labeled cRNAs were mixed and competitively hybridized to human GE 4x44K microarrays (Agilent, G4112F) for $18 \mathrm{~h}$ using an Agilent hybridization kit. Slides were washed according to the manufacturer's protocols and scanned using an Agilent G2565BA scanner. Raw image data were extracted and subjected to standard background subtraction and linear and lowess normalization using Agilent Feature extraction software v9.5. Heatmaps were generated using MATLAB ${ }^{\circledR}$ software (MathWorks Natick, MA, USA). More detailed methods are described in Additional file 14.

\section{Additional material}

Additional file 1: Western blots of whole cell extracts from cell lines expressing FLAG-Brd4, FLAG-HP1 $\beta$ and control cell line (empty vector). Blots were probed with anti-Brd4 (mAb Epitomics, 5716 Burlingame, CA, USA), anti-HP1 $\beta$ (pAb Cell Signaling Technology 2613 Danvers, MA, USA) and $\beta$-actin control (mAb Santa Cruz, sc-81178 Santa Cruz, CA, USA).

Additional file 2: Table of relative PTM abundances determined by quantitative mass spectrometry on histones $\mathrm{H} 3$ and $\mathrm{H} 4$ averaged from three independent ChIP experiments with each Brd and HP1 protein and data from three experiments with HEK293 genomic chromatin.

Additional file 3: Table of relative PTM abundances determined by quantitative mass spectrometry on the histone $\mathrm{H} 4$ peptide (amino acids 4 to 17) averaged from three independent ChIP experiments with each Brd and HP1 protein and data from three experiments with HEK293 genomic chromatin. 
Additional file 4: $P$-values from $t$-tests performed on the fold changes (ChIP/Genomic) from the histone $\mathrm{H} 4$ data presented in Additional file 3. $t$-Tests were performed with data from three independent ChIP experiments for each Brd and HP1 protein and data from three experiments with HEK293 genomic chromatin. P-values were adjusted using the Benjamini-Hochberg correction method to control the false discovery rate (FDR).

Additional file 5: $P$-values from $\boldsymbol{t}$-tests performed on the fold changes (ChIP/Genomic) from the histone data presented in Additional file 2. $t$-Tests were performed with data from three independent ChIP experiments for each Brd and HP1 protein and data from three experiments with HEK293 genomic chromatin. P-values were adjusted using the Benjamini-Hochberg correction method to control the false discovery rate (FDR).

Additional file 6: Table of relative combinatorial PTM abundances determined by quantitative mass spectrometry on the histone H3 peptides (amino acids 9 to 17), (amino acids 18 to 26) and (amino acids 27 to 40 ) averaged from three independent ChIP experiments with each Brd and HP1 protein and data from three experiments with HEK293 genomic chromatin.

Additional file 7: $P$-values from $t$-tests performed on the fold changes (ChIP/Genomic) from the histone $\mathrm{H} 3$ data presented in Additional file 6. $t$-Tests were performed with data from three independent ChIP experiments for each Brd and HP1 protein and data from three experiments with HEK293 genomic chromatin. P-values were adjusted using the Benjamini-Hochberg correction method to control the false discovery rate (FDR)

Additional file 8: Spreadsheets of all promoters bound by the Brd and HP1 proteins. Promoters are ranked by P-values.

Additional file 9: Heatmap of motifs enriched in the HP1 and Brd ChIPs. Lists of consensus sequences (motifs) are found in the matrix used to create the heatmap (Additional file 10)

Additional file 10: Spreadsheets containing matrix used to create the heatmap of motifs enriched in Brd and HP1 ChIPs (Additional file 9)

Additional file 11: Spreadsheets containing Gene Ontology terms enriched in Brd and HP1 ChIPs. Gene Ontology terms are ranked by false discovery rates (FDRs).

Additional file 12: Products from PCR reactions were run on $2 \%$ agarose gels stained with ethidium bromide and visualized on a Gel Doc XR system (BioRad ${ }^{\circledR \circledast}$ Hercules, CA, USA). One half of each $P C R$ reaction was loaded. Gel is labeled corresponding to the templates used for the PCR reactions: control ChIP (beads alone), Brd4 ChIP and ChIP input DNA.

Additional file 13: Western blots of whole cell extracts from Brd4 shRNA knockdown, HP1 $\beta$ shRNA knockdown and control shRNA knockdown cell lines. Blots were probed with anti-Brd4 (mAb Epitomics, 5716), anti-HP1 $\beta$ (pAb Cell Signaling Technology 2613) and $\beta$ actin control (mAb Santa Cruz, sc-81178)

Additional file 14: Supplemental Materials and methods.

\section{Abbreviations}

bp: base pair; ChIP: chromatin immunoprecipitation; ChIP-seq: chromatin immunoprecipitation-high throughput deep sequencing; DamID: DNA adenine methyltransferase identification; nanoLC-MS/MS: nano scale liquid chromatography-tandem mass spectrometry; PCR: polymerase chain reaction; PHD: plant homeodomain; PTM: post-translational modification; qMS: quantitative mass spectrometry; RNA-Seq: RNA-high-throughput deep sequencing; RPKM: reads per kilobase per million mapped reads; shRNA: short hairpin RNA.

\section{Acknowledgements}

Support from a National Science Foundation (NSF) Early Faculty CAREER award, an NIH Innovator award (DP2OD007447) from the Office of the Director, National Institutes of Health, an NSF grant (CBET-0941143) and the
American Society for Mass Spectrometry Research Award to BAG is gratefully acknowledged. PAD is supported by an NIH NRSA F32 Postdoctoral Fellowship. This research was also supported by an NIH/NIGMS P50GM071508 to the Lewis-Sigler Institute at Princeton University (PI D Botstein). BMZ is supported by an NSF graduate fellowship. The work of IC and KZ was supported by the Division of Intramural Research, National Heart, Lung and Blood Institute, National Institutes of Health, USA.

\section{Author details}

'Department of Molecular Biology, Princeton University, 415 Schultz Laboratory, Princeton NJ 08544, USA. ²Systems Biology Center, National Heart, Lung and Blood Institute, National Institutes of Health, Bethesda, MD 20892, USA. 'Epigenetics Program, Department of Biochemistry and Biophysics, Perelman School of Medicine, University of Pennsylvania, 1009 C Stellar-Chance Laboratories, 422 Curie Boulevard, Philadelphia, PA19104, USA. ${ }^{4}$ Department of Chemistry, Princeton University, Princeton NJ 08544, USA. ${ }^{5}$ Quantitative and Computational Biology Program, Princeton University, Princeton, NJ 08544, USA.

\section{Authors' contributions}

GL performed most of the experimental work. IC carried out the analysis of the deep sequencing data. PAD helped analyze the mass spectrometry data. $M A B$ helped with the analysis of microarray data. BMZ assisted with experimental work. $K Z$ and BAG supervised and participated in the design and coordination of the project. GL and BAG wrote the manuscript. All authors read and approved the final manuscript.

\section{Competing interests}

The authors declare that they have no competing interests.

Received: 7 December 2011 Revised: 16 May 2012

Accepted: 16 August 2012 Published: 16 August 2012

\section{References}

1. Kouzarides T: Chromatin modifications and their function. Cell 2007. 128:693-705

2. Meissner A, Mikkelsen TS, Gu H, Wernig M, Hanna J, Sivachenko A, Zhang X, Bernstein BE, Nusbaum C, Jaffe DB, Jaenisch R, Lander ES: Genome-scale DNA methylation maps of pluripotent and differentiated cells. Nature 2008, 454:766-770

3. Wang Z, Zang C, Rosenfeld JA, Schones DE, Barski A, Cuddapah S, Cui K, Roh TY, Peng W, Zhang MQ, Zhao K: Combinatorial patterns of histone acetylations and methylations in the human genome. Nat Genet 2008, 40:897-903.

4. Barski A, Cuddapah S, Cui K, Roh TY, Schones DE, Wang Z, Wei G, Chepelev I, Zhao K: High-resolution profiling of histone methylations in the human genome. Cell 2007, 129:823-837.

5. Hebbes TR, Thorne AW, Crane-Robinson C: A direct link between core histone acetylation and transcriptionally active chromatin. EMBO J 1988 7:1395-1402

6. Chang YL, King B, Lin SC, Kennison JA, Huang DH: A double-bromodomain protein, FSH-S, activates the homeotic gene ultrabithorax through a critical promoter-proximal region. Mol Cell Biol 2007, 27:5486-5498.

7. Florence B, Faller DV: You bet-cha: a novel family of transcriptional regulators. Front Biosci 2001, 6:D1008-1018.

8. LeRoy G, Rickards B, Flint SJ: The double bromodomain proteins Brd2 and Brd3 couple histone acetylation to transcription. Mol Cell 2008, 30:51-60.

9. Nicodeme $E$, Jeffrey $K L$, Schaefer $U$, Beinke $S$, Dewell $S$, Chung CW, Chandwani R, Marazzi I, Wilson P, Coste H, White J, Kirilovsky J, Rice CM, Lora JM, Prinjha RK, Lee K, Tarakhovsky A: Suppression of inflammation by a synthetic histone mimic. Nature 2010, 468:1119-1123.

10. Denis GV, Vaziri C, Guo N, Faller DV: RING3 kinase transactivates promoters of cell cycle regulatory genes through E2F. Cell Growth Differ 2000, 11:417-424

11. Filippakopoulos P, Qi J, Picaud S, Shen Y, Smith WB, Fedorov O, Morse EM, Keates T, Hickman TT, Felletar I, Philpott M, Muro S, McKeown MR, Wang Y, Christie AL, West N, Cameron MJ, Schwartz B, Heightman TD, La Thangue N, French CA, Wiest O, Kung AL, Knapp S, Bradner JE: Selective inhibition of BET bromodomains. Nature 2010, 468:1067-1073.

12. Jacobs SA, Khorasanizadeh S: Structure of HP1 chromodomain bound to a lysine 9-methylated histone H3 tail. Science 2002, 295:2080-2083. 
13. Levine SS, King IF, Kingston RE: Division of labor in polycomb group repression. Trends Biochem Sci 2004, 29:478-485.

14. Nielsen AL, Oulad-Abdelghani M, Ortiz JA, Remboutsika E, Chambon P, Losson R: Heterochromatin formation in mammalian cells: interaction between histones and HP1 proteins. Mol Cell 2001, 7:729-739.

15. Dialynas GK, Terjung S, Brown JP, Aucott RL, Baron-Luhr B, Singh PB, Georgatos SD: Plasticity of HP1 proteins in mammalian cells. J Cell Sci 2007, 120:3415-3424.

16. Cammas $F$, Janoshazi $A$, Lerouge $T$, Losson R: Dynamic and selective interactions of the transcriptional corepressor TIF1 beta with the heterochromatin protein HP1 isotypes during cell differentiation. Differentiation 2007, 75:627-637.

17. Bartova E, Pachernik J, Kozubik A, Kozubek S: Differentiation-specific association of HP1alpha and HP1beta with chromocentres is correlated with clustering of TIF1beta at these sites. Histochem Cell Biol 2007, 127:375-388.

18. Plazas-Mayorca MD, Zee BM, Young NL, Fingerman IM, LeRoy G, Briggs SD, Garcia BA: One-pot shotgun quantitative mass spectrometry characterization of histones. J Proteome Res 2009, 8:5367-5374.

19. Umehara T, Nakamura Y, Jang MK, Nakano K, Tanaka A, Ozato K, Padmanabhan B, Yokoyama S: Structural basis for acetylated histone $\mathrm{H} 4$ recognition by the human BRD2 bromodomain. J Biol Chem 2010, 285:7610-7618

20. Liu Y, Wang $X$, Zhang J, Huang H, Ding B, Wu J, Shi Y: Structural basis and binding properties of the second bromodomain of Brd4 with acetylated histone tails. Biochemistry 2008, 47:6403-6417.

21. Lachner M, O'Carroll D, Rea S, Mechtler K, Jenuwein T: Methylation of histone H3 lysine 9 creates a binding site for HP1 proteins. Nature 2001, 410:116-120.

22. Liu H, Galka M, Iberg A, Wang Z, Li L, Voss C, Jiang X, Lajoie G, Huang Z, Bedford MT, Li SS: Systematic identification of methyllysine-driven interactions for histone and nonhistone targets. J Proteome Res 2010, 9:5827-5836

23. Jin $Q$, Yu LR, Wang $L$, Zhang $Z$, Kasper $L H$, Lee JE, Wang $C$, Brindle $P K$ Dent SY, Ge K: Distinct roles of GCN5/PCAF-mediated H3K9ac and CBP/ p300-mediated H3K18/27ac in nuclear receptor transactivation. EMBO J 2011, 30:249-262.

24. van Oevelen CJ, van Teeffelen HA, van Werven FJ, Timmers HT: Snf1pdependent Spt-Ada-Gcn5-acetyltransferase (SAGA) recruitment and chromatin remodeling activities on the HXT2 and HXT4 promoters. J Biol Chem 2006, 281:4523-4531.

25. Wang D, Xia X, Weiss RE, Refetoff S, Yen PM: Distinct and histone-specific modifications mediate positive versus negative transcriptional regulation of TSHalpha promoter. PLOS One 2010, 5:e9853.

26. Margueron R, Li G, Sarma K, Blais A, Zavadil J, Woodcock CL, Dynlacht BD, Reinberg D: Ezh1 and Ezh2 maintain repressive chromatin through different mechanisms. Mol Cell 2008, 32:503-518.

27. Bernstein E, Duncan EM, Masui O, Gil J, Heard E, Allis CD: Mouse polycomb proteins bind differentially to methylated histone $\mathrm{H} 3$ and RNA and are enriched in facultative heterochromatin. Mol Cell Biol 2006, 26:2560-2569.

28. Plazas-Mayorca MD, Bloom JS, Zeissler U, Leroy G, Young NL, DiMaggio PA, Krugylak L, Schneider R, Garcia BA: Quantitative proteomics reveals direct and indirect alterations in the histone code following methyltransferase knockdown. Mol Biosyst 2010, 6:1719-1729.

29. Bernstein BE, Mikkelsen TS, Xie X, Kamal M, Huebert DJ, Cuff J, Fry B, Meissner A, Wernig M, Plath K, Jaenisch R, Wagschal A, Feil R, Schreiber SL, Lander ES: A bivalent chromatin structure marks key developmental genes in embryonic stem cells. Cell 2006, 125:315-326.

30. Brock HW, Hodgson JW, Petruk S, Mazo A: Regulatory noncoding RNAs at Hox loci. Biochem Cell Biol 2009, 87:27-34.

31. Lewis EB: A gene complex controlling segmentation in Drosophila. Nature 1978, 276:565-570.

32. Ingham PW: trithorax and the regulation of homeotic gene expression in Drosophila: a historical perspective. Int J Dev Biol 1998, 42:423-429.

33. Mallo M, Wellik DM, Deschamps J: Hox genes and regional patterning of the vertebrate body plan. Dev Biol 2010, 344:7-15.

34. Petruk S, Sedkov Y, Smith S, Tillib S, Kraevski V, Nakamura T, Canaani E, Croce CM, Mazo A: Trithorax and dCBP acting in a complex to maintain expression of a homeotic gene. Science 2001, 294:1331-1334

35. Yokoyama A, Wang Z, Wysocka J, Sanyal M, Aufiero DJ, Kitabayashi I, Herr W, Cleary ML: Leukemia proto-oncoprotein MLL forms a SET1-like histone methyltransferase complex with menin to regulate Hox gene expression. Mol Cell Biol 2004, 24:5639-5649.

36. Papoulas O, Beek SJ, Moseley SL, McCallum CM, Sarte M, Shearn A, Tamkun JW: The Drosophila trithorax group proteins BRM, ASH1 and ASH2 are subunits of distinct protein complexes. Development 1998, 125:3955-3966.

37. Shannon M, Ashworth LK, Mucenski ML, Lamerdin JE, Branscomb E, Stubbs $L$ : Comparative analysis of a conserved zinc finger gene cluster on human chromosome 19q and mouse chromosome 7. Genomics 1996, 33:112-120.

38. Vogel MJ, Guelen L, de Wit E, Peric-Hupkes D, Loden M, Talhout W, Feenstra M, Abbas B, Classen AK, van Steensel B: Human heterochromatin proteins form large domains containing KRAB-ZNF genes. Genome Res 2006, 16:1493-1504.

39. Yang Z, Yik JH, Chen R, He N, Jang MK, Ozato K, Zhou Q: Recruitment of PTEFb for stimulation of transcriptional elongation by the bromodomain protein Brd4. Mol Cell 2005, 19:535-545.

40. Atkinson SP, Koch CM, Clelland GK, Willcox S, Fowler JC, Stewart R, Lako M, Dunham I, Armstrong L: Epigenetic marking prepares the human HOXA cluster for activation during differentiation of pluripotent cells. Stem Cells 2008, 26:1174-1185.

41. Moving AHEAD with an international human epigenome project.. Nature 2008, 454:711-715.

42. Kashyap V, Gudas LJ, Brenet F, Funk P, Viale A, Scandura JM: Epigenomic reorganization of the clustered Hox genes in embryonic stem cells induced by retinoic acid. J Biol Chem 2011, 286:3250-3260.

43. Ku M, Koche RP, Rheinbay E, Mendenhall EM, Endoh M, Mikkelsen TS, Presser A, Nusbaum C, Xie X, Chi AS, Adi M, Kasif S, Ptaszek LM, Cowan CA, Lander ES, Bernstein BE: Genomewide analysis of PRC1 and PRC2 occupancy identifies two classes of bivalent domains. PLoS Genet 2008, 4: e1000242.

44. LeRoy G, Weston JT, Zee BM, Young NL, Plazas-Mayorca MD, Garcia BA: Heterochromatin protein 1 is extensively decorated with histone codelike post-translational modifications. Mol Cell Proteomics 2009, 8:2432-2442.

45. DiMaggio PA, Young NL, Baliban RC, Garcia BA, Floudas CA: A mixed integer linear optimization framework for the identification and quantification of targeted post-translational modifications of highly modified proteins using multiplexed electron transfer dissociation tandem mass spectrometry. Mol Cell Proteomics 2009, 8:2527-2543.

46. Proteome Commons using the following Tranche hash: ihSXDmjmtLL5W8cn2t5 +NkkENajlU9T0D9hLPkJ3nvLiF9MzW0FIgwyjbQIJXUUMiMiSWB9zK/ 7hUyqKSt50ViC/IYgAAAAAAAABtg==.. [https://proteomecommons.org/].

47. Sultan M, Schulz MH, Richard $H$, Magen A, Klingenhoff $A$, Scherf $M$, Seifert M, Borodina T, Soldatov A, Parkhomchuk D, Schmidt D, O'Keeffe S, Haas S, Vingron M, Lehrach H, Yaspo ML: A global view of gene activity and alternative splicing by deep sequencing of the human transcriptome. Science 2008, 321:956-960.

48. Langmead B, Trapnell C, Pop M, Salzberg SL: Ultrafast and memoryefficient alignment of short DNA sequences to the human genome. Genome Biol 2009, 10:R25.

49. Zang C, Schones DE, Zeng C, Cui K, Zhao K, Peng W: A clustering approach for identification of enriched domains from histone modification ChIP-Seq data. Bioinformatics 2009, 25:1952-1958.

50. Mortazavi A, Williams BA, McCue K, Schaeffer L, Wold B: Mapping and quantifying mammalian transcriptomes by RNA-Seq. Nat Methods 2008, 5:621-628

51. UCSC Genome Browser.. [http://genome.ucsc.edu/].

52. Gene Expression Omnibus.. [http://www.ncbi.nlm.nih.gov/geo].

\section{doi:10.1186/gb-2012-13-8-r68}

Cite this article as: LeRoy et al:: Proteogenomic characterization and mapping of nucleosomes decoded by Brd and HP1 proteins. Genome Biology 2012 13:R68. 